\title{
An evaluation of $\left|V_{u s}\right|$ and precise tests of the Standard Model from world data on leptonic and semileptonic kaon decays
}

\author{
M. Antonelli' ${ }^{2}$, V. Cirigliano 3 , G. Isidori ${ }^{2}$, F. Mescia ${ }^{1}$, M. Moulson ${ }^{2}$, H. Neufeld ${ }^{8}$, E. Passemar ${ }^{7}$, M. Palutan ${ }^{2}$, \\ B. Sciascia ${ }^{2}$, M. Sozzi ${ }^{5}$, R. Wanke ${ }^{4}$, O.P. Yushchenko ${ }^{6}$, for the FlaviaNet Working Group on Kaon Decays ${ }^{9}$ \\ 1 Dep. ECM and ICC, Universitat de Barcelona, 08028 Barcelona, Spain \\ 2 Laboratori Nazionali di Frascati dell'INFN, 00044 Frascati RM, Italy \\ 3 Theoretical Division, Los Alamos National Laboratory, Los Alamos, NM, 87545, USA \\ 4 Institut für Physik, Universität Mainz, 55099 Mainz, Germany \\ 5 Dipartimento di Fisica, Università di Pisa e Sezione dell'INFN di Pisa, 56100 Pisa, Italy \\ Institute for High Energy Physics, 142284 Protvino, Russia \\ 7 Departament de Física Teòrica, IFIC, Universitat de València - CSIC, 46071 València, Spain \\ 8 Fakultät für Physik, Universität Wien, 1090 Wien, Austria \\ 9 http://www.lnf.infn.it/wg/vus
}

Received: date / Revised version: date

\begin{abstract}
We present a global analysis of leptonic and semileptonic kaon decay data, including all recent results published by the BNL-E865, KLOE, KTeV, ISTRA+ and NA48 experiments. This analysis, in conjunction with precise lattice calculations of the hadronic matrix elements now available, leads to a very precise determination of $\left|V_{u s}\right|$ and allows us to perform several stringent tests of the Standard Model.
\end{abstract}

PACS. 13.20.Eb Decays of $K$ mesons

\section{Introduction}

Within the Standard Model (SM), leptonic and semileptonic kaon decays can be used to obtain the most accurate determination of the magnitude of the element $V_{u s}$ of the Cabibbo-Kobayashi-Maskawa (CKM) matrix [1,2]. A detailed analysis of these processes potentially also provides stringent constraints on new physics scenarios: while within the SM, all $d^{i} \rightarrow u^{j} \ell \nu$ transitions are ruled by the same CKM coupling $V_{j i}$ (satisfying the unitarity condition $\sum_{k}\left|V_{j k}\right|^{2}=1$ ), and $G_{F}$ is the same coupling that governs muon decay, this is not necessarily true beyond the SM. New bounds on violations of CKM unitarity and lepton universality and deviations from the $V-A$ structure translate into significant constraints on various newphysics scenarios. Alternately, such tests may eventually turn up evidence of new physics.

In the case of leptonic and semileptonic kaon decays, these tests are particularly significant given (i) the large amount of data recently collected by several experiments, (ii) the substantial progress recently made in evaluating the corresponding hadronic matrix elements from lattice QCD, and (iii) the precise analytic calculations of radiative corrections and isospin-breaking effects recently performed within chiral perturbation theory (ChPT), the low-energy effective theory of QCD. This progress on both

Correspondence to: Matthew.Moulson@lnf.infn.it the experimental and the theoretical sides allows for unique tests of the SM that probe very high energy scales.

An illustration of the importance of semileptonic kaon decays in testing the SM is provided by the unitarity relation

$$
\left|V_{u d}\right|^{2}+\left|V_{u s}\right|^{2}+\left|V_{u b}\right|^{2}=1+\Delta_{\mathrm{CKM}} .
$$

Here the $V_{j i}$ are the CKM elements as determined from the various $d^{i} \rightarrow u^{j}$ processes, where the value of $G_{F}$ is determined from the muon life time: $G_{\mu}=1.166371(6) \times$ $10^{-5} \mathrm{GeV}^{-2}$ [3]. $\Delta_{\mathrm{CKM}}$ parameterizes possible deviations from the SM induced by dimension-six operators, contributing either to muon decay or to $d^{i} \rightarrow u^{j}$ transitions. As we will show in the following, the present accuracy on $\left|V_{u s}\right|$ allows us to set bounds on $\Delta_{\mathrm{CKM}}$ around $0.1 \%$, which translate into bounds on the effective scale of new physics on the order of $10 \mathrm{TeV}$.

A detailed analysis of precise tests of the SM with leptonic and semileptonic kaon decays has already been presented in Ref. 4. However, the significant progress on both the experimental and theoretical sides has motivated us to perform an updated analysis with three major areas of emphasis: (i) the determination of $\left|V_{u s}\right|$ from experimental data, with and without imposing CKM unitarity; (ii) the comparison between the values of $\left|V_{u s}\right|$ obtained from data on $K \rightarrow \pi \ell \nu\left(K_{\ell 3}\right)$ and $K \rightarrow \mu \nu\left(K_{\mu 2}\right)$ decays and the corresponding constraints on deviations from the 
$V-A$ structure of the charged current; (iii) tests of lepton universality in $K_{\ell 3}$ decays.

To carry out this analysis, values are needed for the hadronic constants $f_{K} / f_{\pi}$ and $f_{+}(0)$, as discussed in Sects. 2.1.1 and 2.2.1. These values are obtained using lattice QCD, and various determinations have been performed. The lattice QCD community, as represented by the FlaviaNet Lattice Averaging Group (FLAG) [5] 7], is progressing towards convergence on a set of reference values, but in the meantime, for the purposes of this work, we are led to propose our own. The criteria we have applied in averaging lattice QCD results are motivated, but do not represent the only set of possible choices. Our adoption of these values is intended to illustrate what precision can be obtained in testing the SM, given current experimental and theoretical results. In particular, wherever possible we quote results for quantities such as $\left|V_{u s}\right| f_{+}(0)$ or $\left|V_{u s} / V_{u d}\right| \times f_{K} / f_{\pi}$, which are independent of lattice inputs and ready for use as new lattice results become available.

This paper is organized as follows. The phenomenological framework needed to describe $K_{\ell 3}$ and $K_{\mu 2}$ decays within and beyond the SM is briefly reviewed in Sect. 2, The experimental data is reviewed and combined in Sect. 3. The results are presented and interpreted in Sect. 4 .

\section{Phenomenological framework}

\section{$2.1 K_{\ell 2}$ rates in the Standard Model}

Within the SM, the ratio of photon-inclusive $K^{ \pm} \rightarrow \ell^{ \pm} \nu$ $\left(K_{\ell 2(\gamma)}^{ \pm}\right)$to $\pi^{ \pm} \rightarrow \ell^{ \pm} \nu\left(\pi_{\ell 2(\gamma)}^{ \pm}\right)$decay rates can be written as 8,9

$$
\frac{\Gamma_{K_{\ell 2}}}{\Gamma_{\pi_{\ell 2}}}=\frac{\left|V_{u s}\right|^{2}}{\left|V_{u d}\right|^{2}} \frac{f_{K}^{2}}{f_{\pi}^{2}} \frac{m_{K}\left(1-m_{\ell}^{2} / m_{K}^{2}\right)^{2}}{m_{\pi}\left(1-m_{\ell}^{2} / m_{\pi}^{2}\right)^{2}}\left(1+\delta_{\mathrm{EM}}\right),
$$

where $f_{K}$ and $f_{\pi}$ are the kaon and pion decay constants, and $\delta_{\mathrm{EM}}$ denotes the effect of long-distance electromagnetic corrections. Short-distance radiative effects are universal and cancel from the ratio. For pointlike kaons and pions, the long-distance electromagnetic corrections depend only on the particle masses. The dominant uncertainty on $\delta_{\mathrm{EM}}$ comes from terms depending on the hadronic structure. Most analyses to date make use of the results of Refs. 10 and 11, which were computed in a model of hadronic structure assuming BreitWigner form factors for the low-lying vector resonances in order to handle the scale matching. These results give $\delta_{\mathrm{EM}}=-0.0070(35)$ (see, e.g., Ref. 12). Using chiral perturbation theory [13,9] it has been shown that to leading nontrivial orden $1 \mathcal{O}\left(e^{2} p^{2}\right)$, the structure-dependent correc-

\footnotetext{
${ }^{1}$ In ChPT, physical amplitudes are systematically expanded in powers of the external momenta of pseudo-Goldstone bosons $(\pi, K, \eta)$ and quark masses. When including electromagnetic corrections, the power counting is in $e^{2 m}\left(p / 4 \pi f_{\pi}\right)^{2 n}$. Powers of the quark masses count as two powers of the external momenta $\left(\mathcal{O}\left(p^{2}\right)=\mathcal{O}\left(m_{q}\right)\right)$.
}

tions to $\delta_{\mathrm{EM}}$ can be expressed in terms of the electromagnetic pion mass splitting. With the relative theoretical uncertainty estimated at $25 \%$ to account for $\mathcal{O}\left(e^{2} p^{4}\right)$ effects suppressed by chiral power counting, one obtains

$$
\delta_{\mathrm{EM}}=-0.0070(18)
$$

With experimental measurements of the inclusive $K_{\ell 2}$ and $\pi_{\ell 2}$ decay rates and precise knowledge of the radiative corrections, Eq. (2) can be used to obtain the value of the ratio

$$
\left|\frac{V_{u s}}{V_{u d}}\right|^{2} \frac{f_{K}^{2}}{f_{\pi}^{2}}
$$

\subsubsection{Theoretical determination of $f_{K} / f_{\pi}$}

To experimentally constrain the ratio $\left|V_{u s} / V_{u d}\right|$, or ultimately, the value of $\left|V_{u s}\right|$ itself, a precise estimate of the ratio of decay constants $f_{K} / f_{\pi}$ is needed. The analytic evaluation of this ratio within ChPT at $\mathcal{O}\left(p^{4}\right)$ depends on unknown low-energy constants (LECs) and thus cannot be predicted with high accuracy. Consequently, precise evaluations of $f_{K} / f_{\pi}$ are obtained only from lattice QCD. However, ChPT still provides useful information on this ratio: the $\mathrm{SU}(3)$ breaking of $f_{K} / f_{\pi}$ is linear in $m_{K}^{2}-m_{\pi}^{2} \propto m_{s}-m_{u}$ and thus potentially large. For lattice determinations of $f_{K} / f_{\pi}$, this implies that the use of very light pions is essential to obtain reliable results.

During the last few years, new simulations with $N_{F}=$ 2, $N_{F}=2+1$, and $N_{F}=2+1+1$ flavors of dynamical quarks have been performed by several groups using many different lattice QCD formulations, such as staggered (MILC 14]), domain-wall (RBC [15]), overlap (JLQCD [16]), and Wilson-like fermions (BMW [17], CERN-TOV/CLS [18, PACS-CS [19, and ETMC [20]). The fundamental characteristic of these new-generation unquenched studies is that recent technical and conceptual developments 21 have allowed pion masses well below $300 \mathrm{MeV}$ to be reached with large physical volumes $(L$ up to $4 \mathrm{fm}$ ). The PACS-CS [19] collaboration for example has already simulated pions as light as $m_{\pi}=156 \mathrm{MeV}$ for $N_{F}=2+1$ (degenerate $u$ and $d$ quarks) and clover (firstprinciple lattice QCD) fermions. The resulting PACS-CS value, $f_{K} / f_{\pi}=1.189(20)$ [19], however, is still plagued by large uncertainties due to the small simulated volume, $L m_{\pi} \gtrsim 2.3$, with corresponding finite-size effects $\delta_{\mathrm{FSE}}=\exp \left(-L m_{\pi}\right) \approx 10 \%$.

The present status of lattice results for $f_{K} / f_{\pi}[22$, 15, 33, 19,34 37 is summarized in Fig. 1, The lightest pion mass simulated is listed for each determination, together with the smallest lattice spacing used, whenever available. The agreement between the different results is remarkable. The present overall accuracy is about $1 \%$.

Among the results for $f_{K} / f_{\pi}$ in Fig. 1 particularly noteworthy are the $N_{F}=2+1$ studies from BMW [37, MILC '09 31 and HPQCD/UKQCD 35, for which detailed evaluation of the systematic errors (chiral, continuum and infinite-volume limit) have been completed. 


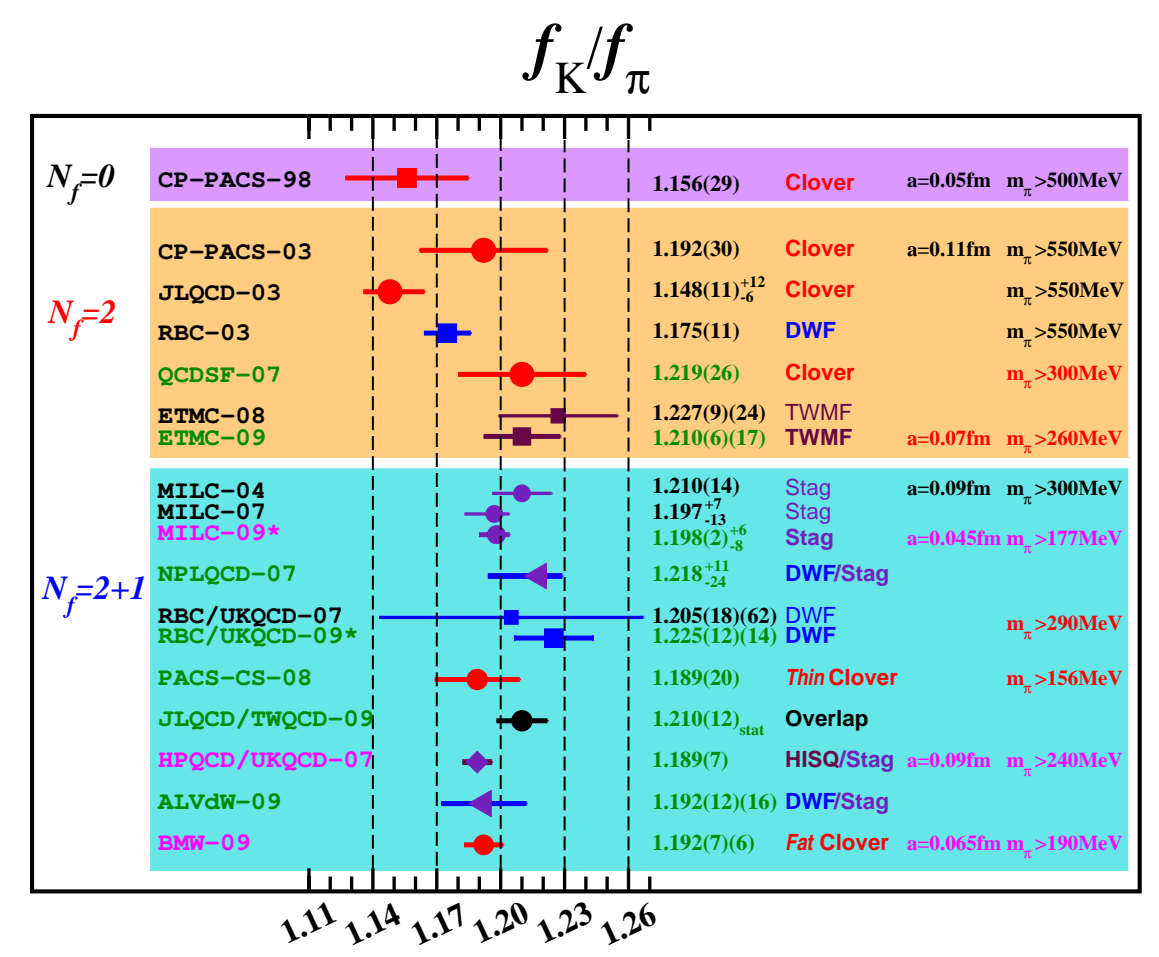

Fig. 1. Summary of lattice determinations of $f_{K} / f_{\pi}$ 22, 32, 15, 33, 19, 34, 37. The smallest lattice spacing $a$ (when available), lightest value of $m_{\pi}$ simulated, and type of lattice fermions used are listed in each case All results for which a value of $a$ is listed have been extrapolated by the authors to the contiunuum limit. Where two types of lattice fermions are listed (e.g. "DWF/Stag"), valence and sea quarks are discretized differently. Unpublished results are indicated by asterisks.

The recent study of $f_{K} / f_{\pi}$ from BMW 37] involves simulations of $N_{F}=2+1$ clover fermions at several volumes with $L m_{\pi} \gtrsim 4$, whereas the continuum limit has been estimated by using three different lattice spacings ( $a \approx 0.065,0.085$, and $0.125 \mathrm{fm}$ ). The BMW value [37] is

$$
f_{K} / f_{\pi}=1.192(7)_{\text {stat }}(6)_{\text {syst }} .
$$

An interesting observation from this study is that the value of $f_{K} / f_{\pi}$ at their lightest pion mass $\left(m_{\pi}=190 \mathrm{MeV}\right.$ at $a=0.085 \mathrm{fm}$ ) is only $2 \%$ below the extrapolated value quoted in Eq. (5). This strengthens the statement that the result is a direct QCD measurement, and not an artificial value cured by an ad hoc fit.

The 2009 MILC result 31, in contrast to the BMW result, was obtained using staggered (AsqTad) fermions. Dynamical AsqTad-staggered ensembles have been generated by MILC since 2002 [29, 30] and now represent an ample data set for the study of lattice systematics featuring six different lattice spacings (ranging from $a=0.18 \mathrm{fm}$ down to $a=0.045 \mathrm{fm})$, light pions $\left(m_{\pi} \gtrsim 177 \mathrm{MeV}\right)$, and large volumes $\left(L m_{\pi} \gtrsim 4\right)$. This MILC activity marked the end of the era of quenched calculations and the start of high-precision lattice QCD with light pions. In their 2009 analysis of $f_{K} / f_{\pi}$ [31, MILC exploits the new lattice ensembles with $a=0.06 \mathrm{fm}\left(m_{\pi} \gtrsim 224 \mathrm{MeV}\right)$ and $a=0.045 \mathrm{fm}\left(m_{\pi} \gtrsim 324 \mathrm{MeV}\right)$, together with the older $a=0.09 \mathrm{fm}\left(m_{\pi} \gtrsim 180 \mathrm{MeV}\right)$ data set. The resulting value of $f_{K} / f_{\pi} 31$ is

$$
f_{K} / f_{\pi}=1.198(2)_{\text {stat }}\left({ }_{-8}^{+6}\right)_{\text {syst }}=1.197(7),
$$

which is in good agreement with the BMW result (Eq. (5)). (We perform the symmetrization of the total uncertainties in Eq. (6) to facilitate error propagation.) However, the sizable shift with respect to the earlier 2004 MILC result 29, which made use of a coarser lattice and heavier pions than were available in 2009, should be noted. This is a consequence of the sizable systematic errors affecting $f_{K} / f_{\pi}$ : significant shifts in the central values can arise when going to lighter pion masses and the continuum limit (consider also the ETMC results 28, 27 in the $N_{F}=2$ case). For this reason, we consider to be exploratory (although important) the studies of $f_{K} / f_{\pi}$ in which essentially a single lattice spacing is used, including the results shown in Fig. 11 from RBC/UKQCD [33, JLQCD-TWQCD [16, NPLQCD [32, PACS-CS [19, and Ref. [36.

Lastly, for the study from HPQCD/UKQCD 35 the MILC $a=0.15 \mathrm{fm}, 0.12 \mathrm{fm}$, and $0.09 \mathrm{fm}$ ensembles are used; as in the study from MILC, sea quarks are treated using AsqTad staggered fermions. However, in contrast to the MILC approach, valence quarks are described using the Highly-Improved Staggered Quark (HISQ) formulation. The HISQ QCD action [35] is understood to give better lattice scaling - indeed, the MILC Collaboration itself is generating new ensembles with the HISQ action [38. 
The $f_{K} / f_{\pi}$ value from HPQCD/UKQCD [35] is

$$
f_{K} / f_{\pi}=1.189(2)_{\text {stat }}(7)_{\text {syst }} .
$$

This result is in good agreement with the BMW and MILC results. In particular, there is no apparent systematic difference between the results obtained using staggered and clover fermions. This seems to suggest that possible issues associated with the use of staggered fermions (in particular, the rooting issue [39 41]) are not relevant to the determination of $f_{K} / f_{\pi}$, at least at the present level of accuracy.

In order to fully exploit the data set in Fig. 1, we average the results of the analyses from BMW, MILC '09, and HPQCD/UKQCD discussed above (Eqs. (5), (6), and (7)). Since these results are consistent, we calculate the average weighted by the statistical errors on the individual results. This gives our reference central value and its statistical error. To obtain the total error on this average, we assume a systematic error of 0.006 , equal to the smallest systematic error quoted among the three inputs. This is justified on the basis of the agreement between the results. Adding the statistical and systematic errors in quadrature, we obtain

$$
f_{K} / f_{\pi}=1.193(6)
$$

which is quite consistent with all the results in Fig. 1, including those obtained with staggered fermions and from preliminary studies. In the above average, possible correlations between the HPQCD/UKQCD and MILC results due to the use of a common ensemble with $a=0.09 \mathrm{fm}$ has been neglected. However, since the valence quarks are treated differently in these two studies, and the analyses are completely different, any potential correlations are diluted. The above average is consistent with, but has a smaller total uncertainty than, both the average from the most recent Lattice conference [6], $f_{K} / f_{\pi}=1.196(10)$ and the preliminary FLAG result [7], $f_{K} / f_{\pi}=1.190(10)$. As we note in Sect. 1, our use of this average to obtain the results presented in Sects. 4.5 and 4.6 represents a scientific choice. Our value for $\left|V_{u s} / V_{u d}\right| \times f_{K} / f_{\pi}$ (Eq. (55D) may be used with an alternate choice for $f_{K} / f_{\pi}$ to rederive the results of Sects. 4.5 and 4.6, if desired.

Updates from PACS-CS, RBC/UKQCD, and JLQCD, in addition to new results (for example, an $N_{F}=2+1+1$ result from ETMC 42]), have already been announced and will soon improve the present situation.

\section{$2.2 K_{\ell 3}$ rates in the Standard Model}

The $K_{\ell 3}$ decays provide ideal channels for the determination of $\left|V_{u s}\right|$. The starting point of the analysis is the expression for the photon-inclusive $K \rightarrow \pi \ell \nu\left(K_{\ell 3(\gamma)}\right)$ decay rate:

$$
\begin{aligned}
\Gamma_{K_{\ell 3}}= & \frac{G_{F}^{2} m_{K}^{5}}{192 \pi^{3}} C_{K}^{2} S_{\mathrm{EW}}\left(\left|V_{u s}\right| f_{+}^{K^{0} \pi^{-}}(0)\right)^{2} I_{K \ell} \\
& \times\left(1+\delta_{\mathrm{EM}}^{K \ell}+\delta_{\mathrm{SU}(2)}^{K \pi}\right)^{2}
\end{aligned}
$$

where $G_{F}$ is the Fermi constant as determined from muon decays, $S_{E W}=1.0232(3)$ [3, 8] is the short-distance electroweak correction, $C_{K}$ is a Clebsch-Gordan coefficient ( 1 for $K^{0}$ and $1 / \sqrt{2}$ for $K^{ \pm}$decays), $f_{+}^{K^{0} \pi^{-}}(0)$ is the $K^{0} \rightarrow \pi^{-}$vector form factor at zero momentum transfer, and $I_{K \ell}$ is a phase-space integral that is sensitive to the momentum dependence of the form factors. The latter describe the hadronic matrix elements

$$
\begin{aligned}
& \left\langle\pi\left(p_{\pi}\right)\left|\bar{s} \gamma_{\mu} u\right| K\left(p_{K}\right)\right\rangle= \\
& \quad\left(p_{\pi}+p_{K}\right)_{\mu} f_{+}^{K \pi}(t)+\left(p_{K}-p_{\pi}\right)_{\mu} f_{-}^{K \pi}(t),
\end{aligned}
$$

where $t=\left(p_{K}-p_{\pi}\right)^{2}=\left(p_{\ell}+p_{\nu}\right)^{2}$. The vector form factor $f_{+}(t)$ represents the $\mathrm{P}$-wave projection of the crossed channel matrix element $\left\langle 0\left|\bar{s} \gamma^{\mu} u\right| K \pi\right\rangle$. The scalar form factor $f_{0}(t)$ describes the S-wave projection, and in terms of $f_{+}(t)$ and $f_{-}(t)$ reads

$$
f_{0}(t)=f_{+}(t)+\frac{t}{m_{K}^{2}-m_{\pi}^{2}} f_{-}(t) .
$$

By construction, $f_{0}(0)=f_{+}(0)$. Since $f_{+}(0)$ is not directly measurable, it is convenient to factor out $f_{+}^{K^{0} \pi^{-}}(0)$ in Eq. (9) and then normalize the form factors for all channels to $f_{+}^{K^{0} \pi^{-}}(0)$, denoted simply as $f_{+}(0)$ in the following. The normalized form factors are then defined as

$$
\bar{f}_{+}(t)=\frac{f_{+}(t)}{f_{+}(0)}, \bar{f}_{0}(t)=\frac{f_{0}(t)}{f_{+}(0)}, \bar{f}_{+}(0)=\bar{f}_{0}(0)=1 .
$$

Finally, $\delta_{\mathrm{EM}}^{K \ell}$ represents the channel-dependent longdistance EM corrections (Sect. 2.2.2) and $\delta_{\mathrm{SU}(2)}^{K \pi}$ the correction for isospin breaking (Sect. 2.2.3).

To extract $\left|V_{u s}\right|$ from $K_{\ell 3}$ decays using Eq. (9), one must measure one or more photon-inclusive $K_{\ell 3}$ decay rates, compute the phase space integrals from form factor measurements, and make use of theoretical results for $f_{+}(0), \delta_{\mathrm{EM}}^{K \ell}$, and $\delta_{\mathrm{SU}(2)}^{K \pi}$. We discuss the evaluation of these different ingredients in the following.

\subsubsection{Theoretical determination of $f_{+}(0)$}

The vector form factor at zero momentum transfer $f_{+}(0)$ is the most critical hadronic quantity required for the determination of $\left|V_{u s}\right|$ from $K_{\ell 3}$ decays via Eq. (9). By construction, $f_{+}(0)$ is defined in the absence of electromagnetic corrections. More explicitly, $f_{+}(0)$ is defined by the $K^{0} \rightarrow \pi^{-}$matrix element of the vector current, Eq. (10), keeping kaon and pion masses at their physical values.

In this section, we restrict our discussion to the evaluation of $f_{+}(0)$ in the isospin limit2 $\left(m_{u}=m_{d}\right)$. This hadronic quantity cannot be computed in perturbative QCD, but is highly constrained by SU(3) and chiral symmetry. In the chiral limit and, more generally, in the $\mathrm{SU}(3)$

\footnotetext{
${ }^{2}$ The choice of the $K^{0} \rightarrow \pi^{-}$form factor as the common normalization is motivated by its smoothness in the $m_{u}=m_{d}$ limit (see Sect. 2.2.3).
} 
limit $\left(m_{u}=m_{d}=m_{s}\right)$ the conservation of the vector current implies $f_{+}(0)=1$. Expanding around the chiral limit in powers of light quark masses one can write

$$
f_{+}(0)=1+f_{2}+f_{4}+\ldots
$$

where $f_{n}=\mathcal{O}\left(m_{u, d, s}^{n} /\left(4 \pi f_{\pi}\right)^{n}\right)$, and $f_{2}$ and $f_{4}$ are the next-to-leading order (NLO) and next-to-next-to-leading order (NNLO) corrections in ChPT. The Ademollo-Gatto theorem implies that $\left[f_{+}(0)-1\right]$ is at least of second order in the breaking of $\mathrm{SU}(3)$ or in the expansion in powers of $m_{s}-\hat{m}$, where $\hat{m}=\left(m_{u}+m_{d}\right) / 2$. This also implies that $f_{2}$ is free of $\mathcal{O}\left(p^{4}\right)$ counterterms in ChPT and can be computed with high accuracy: $f_{2}=-0.0227$ [44, 45]. $\Delta f$

Difficulties arise with the calculation of the quantity

$$
\Delta f \equiv f_{4}+f_{6}+\ldots=f_{+}(0)-\left(1+f_{2}\right),
$$

which depends on the LECs of ChPT. The original quarkmodel estimate of Leutwyler and Roos [45] gives $\Delta f=$ $-0.016(8)$ and $f_{+}(0)=0.961(8)$. More recently, analytical calculations have been performed to evaluate the NNLO term $f_{4}$, writing it as

$$
f_{4}=\Delta(\mu)+\left.f_{4}\right|^{\operatorname{loc}}(\mu)
$$

where $\Delta(\mu)$ is the loop contribution, with $\mu$ the renormalization scale, computed in Ref. 46, and $\left.f_{4}\right|^{\text {loc }}(\mu)$ is the $\mathcal{O}\left(p^{6}\right)$ local contribution involving $\mathcal{O}\left(p^{4}\right)$ and unknown $\mathcal{O}\left(p^{6}\right)$ LECs. To estimate the latter term, various approaches have been used, including a quark model [46], dispersion relations [47, and $1 / N_{C}$ estimates [48, 49]. These studies obtain the results $\Delta f=0.001(10),-0.003(11)$, $0.007(12)$, and $0.009(9)$, respectively. $\Delta f$ is found to be compatible with zero from these studies. Relative to the quark-model estimate from Ref. 45, these new results are obtained using more sophisticated techniques and feature better control of systematic uncertainties. The resulting values for $f_{+}(0)$ are summarized in Fig. 2 As can be seen, the uncertainties are no smaller that those from the original estimate of Ref. 45, which illustrates the difficulty of calculating $\Delta f$ to below the $1 \%$ level using analytical methods only.

Figure 2also summarizes present results for $f_{+}(0)$ from lattice QCD 50 56. As can be seen in Fig. 2, lattice results, while in remarkable agreement with the original quark-model estimate of Ref. 45, give somewhat smaller results for $f_{+}(0)$ than do recent analytical calculations.

On the lattice, $f_{+}(0)$ is determined using three-point functions at non-vanishing momenta, whereas $f_{K} / f_{\pi}$ is determined from two-point functions at rest. Because of this additional difficulty, current lattice calculations of $f_{+}(0)$ are less mature than those of $f_{K} / f_{\pi}$. In particular, most results shown in Fig. 2 were obtained with only one lattice spacing and with heavy pion masses. Additionally, only one calculation of $f_{+}(0)$ exists with $N_{F}=2+1$ : that from RBC/UKQCD [55,56]. While BMW, MILC, and HPQCD currently have interesting results for $f_{K} / f_{\pi}$, these groups do not yet have results for $f_{+}(0)$.

Nevertheless, the special chiral properties of $f_{+}(0)$ make it possible to obtain lattice estimates with relative uncertainties comparable to those for $f_{K} / f_{\pi}$. Among the lattice results for $f_{+}(0)$ in Fig. 2 particularly noteworthy are the studies from RBC/UKQCD '07 [55], RBC/UKQCD '10 [56], and ETMC [54]. The corresponding values of $f_{+}(0)$ are respectively

$$
\begin{aligned}
& f_{+}(0)=0.9644(33)_{\text {stat }}(34)_{\text {syst-extr }}(14)_{\text {syst-lat }}, \\
& f_{+}(0)=0.9599(33)_{\text {stat }}\left({ }_{-43}^{+31}\right)_{\text {syst-extr }}(14)_{\text {syst-lat }}, \\
& f_{+}(0)=0.9560(57)_{\text {stat }}(62)_{\text {syst }} .
\end{aligned}
$$

(The two contributions to the systematic errors on the $\mathrm{RBC} / \mathrm{UKQCD}$ results are from extrapolation uncertainties and lattice effects, respectively.) In Ref. 55 and in their update [56, RBC/UKQCD make use of a simulation with $N_{F}=2+1$, but a rather coarse lattice spacing $(a=0.11 \mathrm{fm})$ and a lightest pion mass of $m_{\pi} \approx 330 \mathrm{MeV}$. Even though the use of smaller lattice spacings would be advisable, the corresponding error on the $\mathrm{SU}(3)$ breaking of $f_{+}(0)$ seems to be under control to within the stated systematic uncertainty (see, e.g., discussion in Ref.6). A critical issue is the chiral extrapolation from their points with $m_{\pi} \gtrsim 330 \mathrm{MeV}$. In the new study from RBC/UKQCD [56], there is an attempt to quantify the systematic error from the extrapolation, but a better understanding of the NNLO terms in the chiral expansion 3 as well as additional $N_{F}=2+1$ simulation points at smaller pion masses, are needed to fully address this delicate point.

The recent ETMC study [54] makes use of very light pions, as well as different lattice spacings and volumes. Both $\mathrm{SU}(2)$ and $\mathrm{SU}(3)$ chiral fits are investigated and give compatible results, which is in contrast to the findings of RBC/UKQCD, in which the $\mathrm{SU}(2)$ chiral extrapolation for $f_{+}(0)$ has few points with $m_{\pi} \leq 400 \mathrm{MeV}$ and looks problematic [56. In summary, the ETMC result features a more thorough exploration of systematics than the $\mathrm{RBC} / \mathrm{UKQCD}$ result. However, the ETMC simulation still has $N_{F}=2$, and the final uncertainty on $f_{+}(0)$ is inflated to take into account unknown systematics related to the quenching of the strange quark. At present, many other groups are occupied with finalizing their studies of $f_{K} / f_{\pi}$. As attention returns to $f_{+}(0)$, further progress on understanding these systematics should be possible.

For the numerical analysis in Section 4 we use as our reference number

$$
f_{+}(0)=0.959(5),
$$

which is our symmetrization of the recent RBC/UKQCD result [56. However, we note that this value is fairly representative of the results and spread of values in Eqs. (16). This number is also basically consistent with the average of the $N_{F}=2$ ETMC and $N_{F}=2+1$ RBC/UKQCD results as quoted at the most recent Lattice conference $f_{+}(0)=0.962(5)[6]$.

\subsubsection{Electromagnetic effects in $K_{\ell 3}$ decays}

The results of the most recent calculation 58 of the longdistance electromagnetic corrections to the fully-inclusive

\footnotetext{
${ }^{3}$ Ref. 57 describes exploratory NNLO fits using $\mathrm{RBC} / \mathrm{UKQCD}$ results for $f_{0}(t)$.
} 


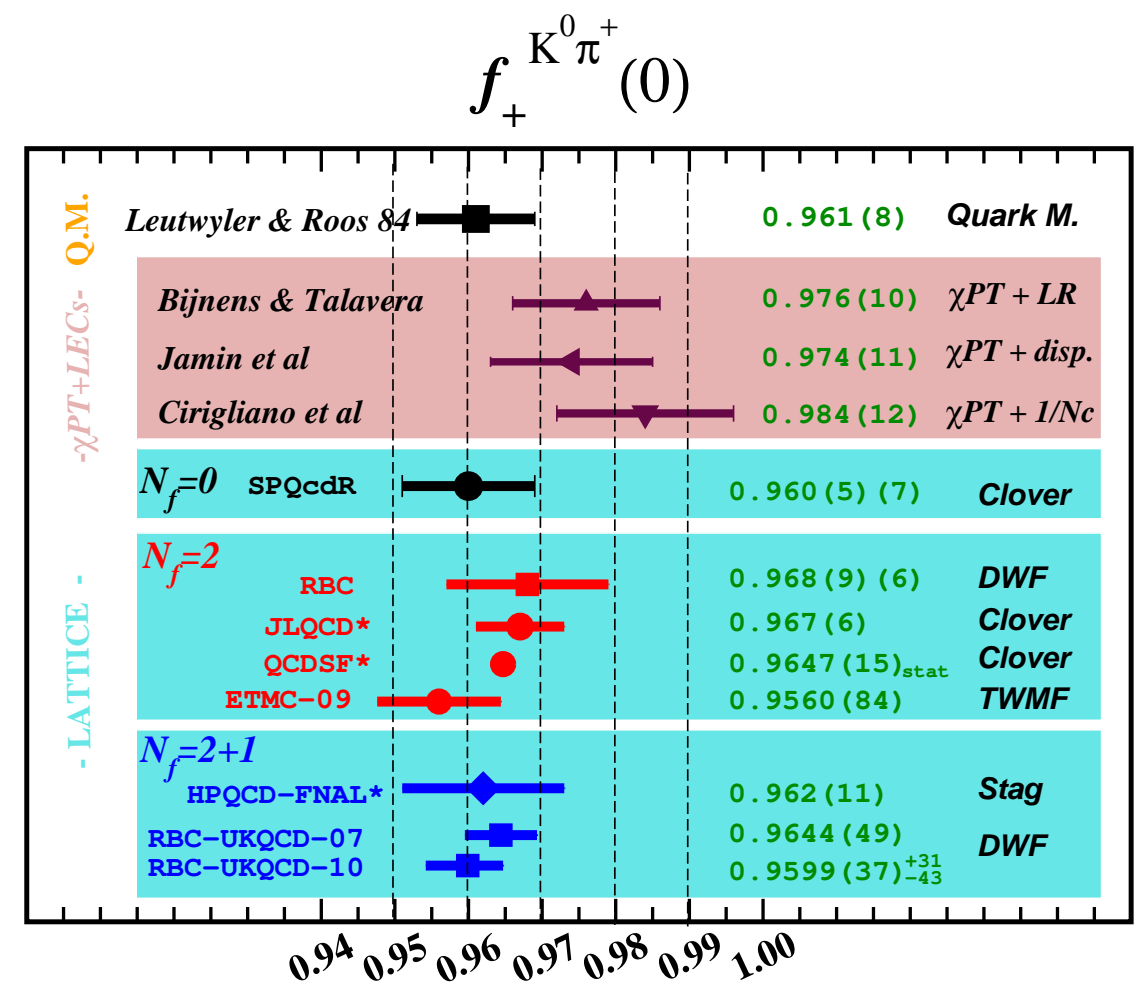

Fig. 2. Present determinations of $f_{+}(0) \equiv f_{+}^{K^{0} \pi^{-}}(0)$ from analytical or semi-analytical approaches [45] 48] and lattice QCD 50 56. Unpublished results are indicated by asterisks.

\begin{tabular}{cc}
\hline \hline Mode & $\delta_{\mathrm{EM}}^{K \ell}(\%)$ \\
\hline$K_{e 3}^{0}$ & $0.495 \pm 0.110$ \\
$K_{e 3}^{ \pm}$ & $0.050 \pm 0.125$ \\
$K_{\mu 3}^{0}$ & $0.700 \pm 0.110$ \\
$K_{\mu 3}^{ \pm}$ & $0.008 \pm 0.125$ \\
\hline \hline
\end{tabular}

Table 1. Electromagnetic corrections to the fully-inclusive $K_{\ell 3(\gamma)}$ rate $[58$.

$K_{\ell 3(\gamma)}$ decay rates for each of the four modes (the $\delta_{\mathrm{EM}}^{K \ell}$ in Eq. (9)) are listed in Table 11. These values were obtained to leading nontrivial order in chiral effective theory, working with a fully-inclusive prescription of real photon emission. For the low-energy electromagnetic couplings appearing in the structure-dependent contributions, the recent determinations of Refs. 59 and 60 were used. The errors in Table 1 are estimates of higher-order contributions that are only partially known. The associated correlation matrix was found to be 58

$$
\left(\begin{array}{r}
+1.000+0.081+0.685-0.147 \\
+1.000-0.147+0.764 \\
+1.000+0.081 \\
+1.000
\end{array}\right)
$$

It is also useful to list the uncertainties on the linear combinations of $\delta_{\mathrm{EM}}^{K \ell}$ that are relevant for lepton-universality and strong isospin-breaking tests (as in Sects.4.2 and 4.3):

$$
\begin{aligned}
\delta_{\mathrm{EM}}^{K^{0} e}-\delta_{\mathrm{EM}}^{K^{0} \mu} & =(-0.205 \pm 0.085) \%, \\
\delta_{\mathrm{EM}}^{K^{ \pm} e}-\delta_{\mathrm{EM}}^{K^{ \pm} \mu} & =(0.042 \pm 0.085) \%, \\
\delta_{\mathrm{EM}}^{K^{ \pm} e}-\delta_{\mathrm{EM}}^{K^{0} e} & =(-0.445 \pm 0.160) \%, \\
\delta_{\mathrm{EM}}^{K^{ \pm} \mu}-\delta_{\mathrm{EM}}^{K^{0} \mu} & =(-0.692 \pm 0.160) \% .
\end{aligned}
$$

The corresponding electromagnetic corrections to the Dalitz plot densities can be found in Ref. 58. It is important to note that the corrections to the Dalitz distributions can be locally large (up to $\sim 10 \%$ ), with considerable cancellations in the integrated electromagnetic corrections. Hence a proper implementation of the electromagnetic corrections in the analysis of the experimental data is essential, in particular for a reliable extraction of the form factor parameters.

\subsubsection{Isospin-breaking corrections in $K_{\ell 3}$ decays}

In Eq. (9), the same quantity $f_{+}(0) \equiv f_{+}^{K^{0} \pi^{-}}(0)$ (form factor at zero momentum transfer) is factored out for all decay channels. The isospin-breaking corrections are then included via the term containing $\delta_{\mathrm{SU}(2)}^{K \pi}$, where

$$
\delta_{\mathrm{SU}(2)}^{K^{0} \pi^{-}}=0, \quad \delta_{\mathrm{SU}(2)}^{K^{+} \pi^{0}}=\frac{f_{+}^{K^{+} \pi^{0}}(0)}{f_{+}^{K^{0} \pi^{-}(0)}}-1 .
$$


This term can be related to the $\pi^{0}-\eta$ mixing [61,49] At leading order $\left(\mathcal{O}\left(p^{2}\right)\right)$ [4]

$$
\delta_{\mathrm{SU}(2)}^{K^{+} \pi^{0}}=\frac{3}{4} \frac{1}{R}, \text { with } R=\frac{m_{s}-\hat{m}}{m_{d}-m_{u}},
$$

while at NLO in the chiral expansion $\left(\mathcal{O}\left(p^{4}\right)\right) 62$

$$
\delta_{\mathrm{SU}(2)}^{K^{+} \pi^{0}}=\frac{3}{4} \frac{1}{R}\left(1+\chi_{p^{4}}+\Delta_{M}+\mathcal{O}\left(m_{q}^{2}\right)\right)
$$

where $\chi_{p^{4}} \approx 0.219$ is an $\mathcal{O}\left(p^{4}\right)$ correction calculable in ChPT [44. $\Delta_{M}$ is a correction (starting at $\left.\mathcal{O}\left(m_{q}\right)\right)$ to the ratio $m_{K}^{2} / m_{\pi}^{2}$ :

$$
\frac{m_{K}^{2}}{m_{\pi}^{2}}=\frac{1}{2}\left(1+\frac{m_{s}}{\hat{m}}\right)\left(1+\Delta_{M}\right)=\frac{Q^{2}}{R}\left(1+\Delta_{M}\right),
$$

where $Q^{2}=\left(m_{s}^{2}-\hat{m}^{2}\right) /\left(m_{d}^{2}-m_{u}^{2}\right)$. Using Eq. (23), Eq. (22) can be rewritten

$$
\delta_{\mathrm{SU}(2)}^{K^{+} \pi^{0}}=\frac{3}{4} \frac{1}{Q^{2}}\left[\frac{m_{K}^{2}}{m_{\pi}^{2}}+\frac{\chi_{p^{4}}}{2}\left(1+\frac{m_{s}}{\hat{m}}\right)\right],
$$

which shows how $\delta_{\mathrm{SU}(2)}^{K^{+} \pi^{0}}$ is essentially determined by the double ratio $Q^{2}$ (the dependence on $m_{s} / \hat{m}$ is suppressed by the smallness of $\chi_{p^{4}}$ ). One can extract $Q^{2}$ from the analysis of the decay $\eta \rightarrow 3 \pi$ or from the kaon mass splitting. A recent analysis using the latter method gives [49]

$$
Q=20.7 \pm 1.2 \text {, }
$$

and thus (using $m_{s} / \hat{m}=24.7 \pm 1.1$ and including $\mathcal{O}\left(e^{2} p^{2}, p^{2}\right)$ corrections 61 to Eq. (24))

$$
\delta_{\mathrm{SU}(2)}^{K^{+} \pi^{0}}=0.029 \pm 0.004 .
$$

This is based on an evaluation of the low-energy electromagnetic couplings 59] leading to a large deviation of Dashen's limit 63]. Note that previous analyses of $\eta \rightarrow 3 \pi$ decays 64 give higher results for $Q$, and hence central values for $\delta_{\mathrm{SU}(2)}^{K^{+} \pi^{0}}$ below the lower edge of the range of values quoted in Eq. (26). New analyses of this decay based on recent data 65] are in progress [66, 67] and should shed light on this issue.

As a final note, the precision reached in the measurement of the $K_{\ell 3}$ decay rates and in the determination of the corrections $\delta_{\mathrm{EM}}^{K \ell}$ allow $\delta_{\mathrm{SU}(2)}^{K^{+} \pi^{0}}$ to be determined directly from data, as discussed in Sect.4.2. By means of Eqs. (22) and (24), the empirical determination of $\delta_{\mathrm{SU}(2)}^{K^{+} \pi^{0}}$ can then be used to derive interesting constraints on the quark mass ratios.

\subsubsection{Parameterization of the form factors}

The last ingredient for the determination of $\left|V_{u s}\right|$ from Eq. (9) is the calculation of the phase space integrals, $I_{K \ell}$

$$
\begin{aligned}
I_{K \ell}=\int_{m_{\ell}^{2}}^{t_{\max }} & d t \frac{1}{m_{K}^{8}} \lambda^{3 / 2}\left(1+\frac{m_{\ell}^{2}}{2 t}\right)\left(1-\frac{m_{\ell}^{2}}{2 t}\right)^{2} \\
& \times\left(\bar{f}_{+}^{2}(t)+\frac{3 m_{\ell}^{2} \Delta_{K \pi}^{2}}{\left(2 t+m_{\ell}^{2}\right) \lambda} \bar{f}_{0}^{2}(t)\right)
\end{aligned}
$$

with $\Delta_{K \pi}=m_{K}^{2}-m_{\pi}^{2}, \lambda=\left[t-\left(m_{K}+m_{\pi}\right)^{2}\right]\left[t-\left(m_{K}-\right.\right.$ $\left.\left.m_{\pi}\right)^{2}\right]$, and $t_{\max }=\left(m_{K}-m_{\pi}\right)^{2}$. In order to calculate the integrals, knowledge is required of the normalized vector and scalar form factors defined in Eq. (12). The form factors can be determined from fits to the measured distributions of the $K_{\ell 3}$ decays in $t$ or some equivalent variable using a given parameterization for the form factors.

Among the different parameterizations proposed in the literature, one can distinguish two classes 68]. Parameterizations based on a systematic mathematical expansion are to date the most widely used. In this class (Class II by the nomenclature of Ref. 68), one finds the Taylor expansion

$$
\begin{aligned}
\bar{f}_{+, 0}^{\text {Taylor }}(t)=1 & +\lambda_{+, 0}^{\prime} \frac{t}{m_{\pi^{ \pm}}^{2}}+\frac{1}{2} \lambda_{+, 0}^{\prime \prime}\left(\frac{t}{m_{\pi^{ \pm}}^{2}}\right)^{2} \\
& +\frac{1}{6} \lambda_{+, 0}^{\prime \prime \prime}\left(\frac{t}{m_{\pi^{ \pm}}^{2}}\right)^{3}+\cdots
\end{aligned}
$$

where $\lambda_{+, 0}^{\prime}$ and $\lambda_{+, 0}^{\prime \prime}$ are the slope and the curvature of the form factors, respectively. Another Class-II parameterization is the so-called $z$-parameterization of Ref. 69 .

In Class-II parameterizations, the parameters describing the higher order terms of the form factor expansion are free to be determined from data. In practice, this additional freedom greatly complicates the use of such parameterizations. As noted in Ref. 70, if a quadratic parameterization is used for both the vector and scalar terms, fits to experimental data will provide no sensitivity to $\lambda_{0}^{\prime \prime}$ because of the strong parameter correlations, especially between $\lambda_{0}^{\prime}$ and $\lambda_{0}^{\prime \prime}$. For this reason, existing power-series fits use a parameterization in $\lambda_{+}^{\prime}, \lambda_{+}^{\prime \prime}$, and $\lambda_{0}$ (see Eq. (28) ). It has been shown in Ref. 71 that in order to describe the form factor shapes accurately in the physical region, one has to go at least up to the second order in the Taylor expansion. This is quantified in Ref. 70, if the same $K_{\mu 3}$ spectrum is fitted using both the linear $\left(\lambda_{0}\right)$ and quadratic $\left(\lambda_{0}^{\prime}, \lambda_{0}^{\prime \prime}\right)$ parameterizations, one typically finds $\lambda_{0} \approx \lambda_{0}^{\prime}+3 \lambda_{0}^{\prime \prime}$. Ignoring the quadratic term increases the phase space integral by about $0.15 \%$. In addition, as discussed below and in Sect. 3.5.3, for tests of low-energy dynamics involving the Callan-Treiman theorem, $\bar{f}_{0}(t)$ must be extrapolated to $t=\Delta_{K \pi} \equiv m_{K}^{2}-m_{\pi}^{2}$, which is well above the endpoint of the physical region in $t$ for $K_{\mu 3}$ decays. A parameterization that accounts for even higher-order terms is therefore desirable.

The parameterizations belonging to Class I circumvent these problems by incorporating additional physical constraints to reduce the number of independent parameters. A typical example is the pole parameterization

$$
\bar{f}_{+, 0}^{\text {pole }}(t)=\frac{M_{V, S}^{2}}{M_{V, S}^{2}-t},
$$

where the dominance of a single resonance is assumed, and the corresponding pole mass $M_{V, S}$ is the only free parameter. While for the vector form factor, a pole parameterization with the dominance of the $K^{*}(892)\left(M_{V} \sim 892\right.$ $\mathrm{MeV}$ ) is in good agreement with the data, for the scalar form factor, there is no such obvious dominance. 
The most interesting and well-motivated parameterizations of Class I are those based on dispersion relations. These are based on the observation that the vector and scalar form factors are analytic functions in the complex $t$-plane, except for a cut along the positive real axis for $t \geq t_{\text {lim }} \equiv\left(m_{K}+m_{\pi}\right)^{2}$, where they develop discontinuities. One can therefore write

$$
\bar{f}_{+, 0}(t)=\frac{1}{\pi} \int_{t_{1 \mathrm{im}}}^{\infty} d s^{\prime} \frac{\operatorname{Im} \bar{f}_{+, 0}\left(s^{\prime}\right)}{\left(s^{\prime}-t-i \epsilon\right)}+\text { subtractions }
$$

where the imaginary part, $\operatorname{Im} \bar{f}_{+, 0}\left(s^{\prime}\right)$, can be determined from data on $K \pi$ scattering, and the ultraviolet component of the integral is absorbed into the (polynomial) subtraction terms. In the vector case, the dispersive parameterization turns out to be numerically very similar to the pole parameterization due to dominant contribution to $\operatorname{Im} \bar{f}_{+}\left(s^{\prime}\right)$ from the $K^{*}(892)$. On the other hand, the dispersive parameterization is particularly useful in the scalar case, where there is no dominant one-particle intermediate state.

In addition to the analyticity constraints, the scalar form factor must satisfy an additional theoretical constraint dictated by chiral symmetry. The Callan-Treiman (CT) theorem 72 implies that the scalar form factor at $t=\Delta_{K \pi} \equiv m_{K}^{2}-m_{\pi}^{2}$ is determined in terms of $f_{K} / f_{\pi}$ and $f_{+}(0)$ up to $\mathcal{O}\left(m_{u, d}\right)$ corrections:

$$
C \equiv \bar{f}_{0}\left(\Delta_{K \pi}\right)=\frac{f_{K}}{f_{\pi}} \frac{1}{f_{+}(0)}+\Delta_{C T} .
$$

The quantity $\Delta_{C T}=\mathcal{O}\left(m_{u, d} / 4 \pi f_{\pi}\right)$ can be evaluated in ChPT. At NLO in the isospin limit 44,

$$
\Delta_{C T}=(-3.5 \pm 8) \times 10^{-3},
$$

where the error is a conservative estimate of the higherorder corrections [73. Results consistent with Eq. (32) from NNLO estimates beyond the isospin limit have been presented in Ref. 49 74, As discussed in Sect. 3.5.3, Eq. (31) provides a useful test of the consistency of the lattice results for $f_{K} / f_{\pi}$ and $f_{+}(0)$ with experimental data on the scalar form factors.

\subsubsection{Dispersive parameterization for the form factors}

Motivated by the existence of the CT theorem, a particularly appealing dispersive parameterization for the scalar form factor has been proposed 71. Two subtractions are performed, one at $t=0$, where by definition $\bar{f}_{0}(0)=1$, and the other at the CT point, $t=\Delta_{K \pi}$. Assuming that the scalar form factor has no zeroes, this leads to

$$
\bar{f}_{0}^{\text {disp }}(t)=\exp \left[\frac{t}{\Delta_{K \pi}}(\ln C-G(t))\right],
$$

with

$$
\begin{aligned}
G(t)= & \frac{\Delta_{K \pi}\left(\Delta_{K \pi}-t\right)}{\pi} \\
& \times \int_{t_{1 \mathrm{im}}}^{\infty} \frac{d s}{s} \frac{\phi_{0}(s)}{\left(s-\Delta_{K \pi}\right)(s-t-i \epsilon)}
\end{aligned}
$$

With this parameterization, the only free parameter to be determined from data is $C$.

The phase $\phi_{0}(s)$ can be identified in the elastic region with the S-wave $(K \pi)_{I=1 / 2}$ scattering phase: performing two subtractions minimizes the contributions from the unknown high-energy phase, which in Ref. 71 is simply and conservatively assumed to lie within the interval $[0,2 \pi)$. The resulting function $G(t)$ in Eq. (34) does not exceed $20 \%$ of the expected value of $\ln C$, while the theoretical uncertainties are at most $10 \%$ of the value of $G(t)$ [1]. The expressions for the leading slope parameters in the Taylor expansion as functions of $\ln C$ are 75

$$
\begin{aligned}
\lambda_{0}^{\prime} & =\frac{m_{\pi}^{2}}{\Delta_{K \pi}}[\ln C-G(0)], \\
\lambda_{0}^{\prime \prime} & =\left(\lambda_{0}^{\prime}\right)^{2}-2 \frac{m_{\pi}^{4}}{\Delta_{K \pi}} G^{\prime}(0), \\
\lambda_{0}^{\prime \prime \prime} & =\left(\lambda_{0}^{\prime}\right)^{3}-6 \frac{m_{\pi}^{4}}{\Delta_{K \pi}} G^{\prime}(0) \lambda_{0}^{\prime}-3 \frac{m_{\pi}^{6}}{\Delta_{K \pi}} G^{\prime \prime}(0),
\end{aligned}
$$

where

$$
\begin{aligned}
G(0) & =0.0398(44), \\
-2 \frac{m_{\pi}^{4}}{\Delta_{K \pi}} G^{\prime}(0) & =4.16(56) \times 10^{-4}, \\
-3 \frac{m_{\pi}^{6}}{\Delta_{K \pi}} G^{\prime \prime}(0) & =2.72(21) \times 10^{-5} .
\end{aligned}
$$

A dispersive representation for the vector form factor can be been built in a similar way [75]. Since there is no equivalent of the $\mathrm{CT}$ theorem in this case, the two subtractions are both performed at $t=0$. The expression analogous to Eq. (33) for the vector form factor is

$$
\bar{f}_{+}^{\text {disp }}(t)=\exp \left[\frac{t}{m_{\pi}^{2}}\left(\Lambda_{+}+H(t)\right)\right]
$$

with

$$
H(t)=\frac{m_{\pi}^{2} t}{\pi} \int_{t_{\mathrm{lim}}}^{\infty} \frac{d s}{s^{2}} \frac{\phi_{+}(s)}{(s-t-i \epsilon)} .
$$

Here the fit parameter is $\Lambda_{+} \equiv m_{\pi}^{2} d \bar{f}_{+}(t) /\left.d t\right|_{t=0}$ and the phase $\phi_{+}(s)$ is derived from $\mathrm{P}$-wave $(K \pi)_{I=1 / 2}$ elastic scattering. As in the case of the scalar form factor, the uncertainty on $H(t)$ has a small influence on the determination of $\Lambda_{+}$. The expressions for the leading slopes in the Taylor expansion as functions of $\Lambda_{+}$are [75].

$$
\begin{aligned}
& \lambda_{+}^{\prime}=\Lambda_{+}, \\
& \lambda_{+}^{\prime \prime}=\left(\lambda_{+}^{\prime}\right)^{2}+2 m_{\pi}^{2} H^{\prime}(0), \\
& \lambda_{+}^{\prime \prime \prime}=\left(\lambda_{+}^{\prime}\right)^{3}+6 m_{\pi}^{2} H^{\prime}(0) \lambda_{+}^{\prime}+3 m_{\pi}^{4} H^{\prime \prime}(0),
\end{aligned}
$$

where

$$
\begin{aligned}
2 m_{\pi}^{2} H^{\prime}(0) & =5.79(97) \times 10^{-4} \\
3 m_{\pi}^{4} H^{\prime \prime}(0) & =2.99(21) \times 10^{-5} .
\end{aligned}
$$

The principal results presented in the following sections are based on the dispersive parameterizations of 
Eqs. (33) and (37). To evaluate the integrals $I_{K \ell}$ from experimental measurements of the parameters $\ln C$ and $\Lambda_{+}$, we use the polynomial expansion given in Appendix A. A detailed discussion of the theoretical uncertainties entering into the dispersive parameterization via the functions $G$ and $H$ can be found in Refs. 71 and 75 .

A final remark concerns the isospin-breaking and electromagnetic corrections. Throughout this work, a universal (isospin-invariant) $t$ dependence is assumed for the normalized form factors in the absence of electromagnetic effects (i.e., we neglect strong isospin-breaking effects in the slopes). Conventionally, the masses appearing in the dispersion integrals are chosen to be the charged pion and neutral kaon masses, while the correct physical masses are used when evaluating the phase space integrals. In principle this is not fully correct. For instance, a different correction $\Delta_{C T}$ should be applied for neutral and charged decays. However, at present, strong isospin-breaking in the slopes can be neglected to well within the experimental errors.

\section{$2.3 K_{\ell 3}$ and $K_{\ell 2}$ beyond the Standard Model}

\subsubsection{Effective Lagrangian for semileptonic decays}

The implications of the precision data on $K_{\ell 2}$ and $K_{\ell 3}$ decays for SM extensions are most conveniently studied within a model-independent effective-theory approach. Within this framework, the most general set of weak-scale dimension-six local operators contributing to the chargedcurrent semileptonic transitions is identified in Ref. 76 under the assumptions that the $\mathrm{SU}(2) \times \mathrm{U}(1)$ symmetry is linearly realized, and that in the neutrino sector, only left-handed neutrinos appear as weak-scale degrees of freedom. The resulting low-scale $(\mu \sim \mathcal{O}(1 \mathrm{GeV}))$ effective Lagrangian governing the semileptonic transitions $d^{j} \rightarrow u^{i} \ell^{-} \bar{\nu}_{\ell}$ for a given lepton flavor $\ell$ involves five operator structures and reads:

$$
\begin{aligned}
\mathcal{L}_{d^{j} \rightarrow u^{i}} & =-2 \sqrt{2} G_{F}^{(0)} V_{i j} \\
\times[ & \left(1+\left[v_{L}\right]_{i j}\right) \bar{\ell}_{L} \gamma_{\mu} \nu_{\ell L} \bar{u}_{L}^{i} \gamma^{\mu} d_{L}^{j} \\
& +\left[v_{R}\right]_{i j} \bar{\ell}_{L} \gamma_{\mu} \nu_{\ell L} \bar{u}_{R}^{i} \gamma^{\mu} d_{R}^{j} \\
& +\left[s_{L}\right]_{i j} \bar{\ell}_{R} \nu_{\ell L} \bar{u}_{R}^{i} d_{L}^{j}+\left[s_{R}\right]_{i j} \bar{\ell}_{R} \nu_{\ell L} \bar{u}_{L}^{i} d_{R}^{j} \\
& \left.+\left[t_{L}\right]_{i j} \bar{\ell}_{R} \sigma_{\mu \nu} \nu_{\ell L} \bar{u}_{R}^{i} \sigma^{\mu \nu} d_{L}^{j}\right] \quad+\text { h.c. },
\end{aligned}
$$

where $G_{F}^{(0)} / \sqrt{2}=g^{2} /\left(8 m_{W}^{2}\right)$. The effective couplings $v_{L, R}$, $s_{L, R}$, and $t_{L}$ encode information on interactions beyond the SM and are of order $v^{2} / \Lambda^{2}$, where $v$ is the SM Higgs vacuum expectation value and $\Lambda$ is the new physics scale. The coupling $v_{L}$ receives contributions from three gaugeinvariant weak-scale operators (gauge boson-quark vertex correction, gauge boson-lepton vertex correction and contact four-fermion) while the other couplings are in oneto-one correspondence with gauge-invariant four-fermion operators at the weak scale.
In general, the effective couplings carry flavor indices and the operators considered here contribute to flavorchanging neutral current (FCNC) processes (this is made explicit by writing the operators in $\mathrm{SU}(2)_{\mathrm{L}}$ gauge-invariant form at the weak scale). In order to avoid the strong constraints from FCNC, it is convenient to classify the operators according to their behavior under the $\mathrm{U}(3)^{5}$ flavor symmetry of the SM gauge Lagrangian and organize the discussion in terms of perturbations around the $\mathrm{U}(3)^{5}$ symmetric limit. In practice, we will assume that the underlying $\mathrm{TeV}$ scale new physics has an approximate $\mathrm{U}(3)^{5}$ invariance. This can be achieved if flavor breaking is suppressed by a mechanism such as Minimal Flavor Violation (MFV) 77 81 or by the hierarchy $\Lambda_{\text {flavor }} \gg 1 \mathrm{TeV}$.

\subsubsection{Phenomenology in the $\mathrm{U}(3)^{5}$ limit}

We start our discussion by assuming dominance of the $\mathrm{U}(3)^{5}$-invariant operators. These are not constrained by FCNC and can have a relatively low effective scale $\Lambda$. Moreover, these operators contribute to a number of precision electroweak tests. Therefore, with this analysis one can assess the interplay and relative strength of low-energy charged-current processes versus other observables (from low-energy to the $Z$ pole).

In the $\mathrm{U}(3)^{5}$ limit, the phenomenology of chargedcurrent processes is greatly simplified [76]: only the SM operators (proportional to $1+v_{L}$ ) survive in the effective Lagrangians both for semileptonic decays (Eq. (41)) and for muon decay, which process is used to determine the Fermi constant. The effects of new physics can therefore be encoded into shifts in the values of the effective Fermi constants for semileptonic and muon decay:

$$
\begin{aligned}
G_{F}^{\mathrm{SL}} & =\left(G_{F}\right)^{(0)}\left(1+v_{L}\right), \\
G_{F}^{\mu} & =\left(G_{F}\right)^{(0)}\left(1+\tilde{v}_{L}\right),
\end{aligned}
$$

allowing for different values of $v_{L}$ in semileptonic and muon decays. The values of the CKM elements $V_{i j}^{\text {phenom }}$ as determined from semileptonic decays are affected by both these shifts, because semileptonic transitions are normalized to the Fermi constant $G_{F}^{\mu}$ as determined from muon decay. In fact, one has

$$
V_{i j}^{\text {phenom }}=V_{i j} \frac{G_{F}^{\mathrm{SL}}}{G_{F}^{\mu}}=V_{i j}\left(1+v_{L}-\tilde{v}_{L}\right) .
$$

In the $\mathrm{U}(3)^{5}$ limit, then, a common shift affects all of the $V_{i j}$ (as determined from all channels: vector, axial, etc.), and the only way to expose contributions from new physics is to construct universality tests in which the absolute normalization of the $V_{i j}$ matters. For light-quark transitions, this involves checking that the first row of the CKM matrix is a vector of unit length. Therefore, one is led to define:

$$
\begin{aligned}
& \Delta_{\mathrm{CKM}} \equiv\left|V_{u d}^{\text {phenom }}\right|^{2}+\left|V_{u s}^{\text {phenom }}\right|^{2}+\left|V_{u b}^{\text {phenom }}\right|^{2}-1,(45) \\
& \hline \text { I.e., the freedom to perform U(3) transformations in family } \\
& \text { space for each of the five fermionic gauge multiplets: } Q_{L}= \\
& \left(u_{L}, d_{L}\right), u_{R}, d_{R}, L_{L}=\left(\nu_{L}, e_{L}\right), e_{R} .
\end{aligned}
$$


in terms of the $V_{i j}^{\text {phenom }}$ determined from semileptonic transitions using the standard procedure. The new-physics contributions to $\Delta_{\mathrm{CKM}}$ involve four weak-scale gaugeinvariant local operators ( $\varphi$ denotes the SM Higgs doublet),

$$
\begin{aligned}
& O_{l l}^{(3)}=\frac{1}{2}\left(\bar{L}_{L} \gamma^{\mu} \sigma^{a} L_{L}\right)\left(\bar{L}_{L} \gamma_{\mu} \sigma^{a} L_{L}\right), \\
& O_{l q}^{(3)}=\left(\bar{L}_{L} \gamma^{\mu} \sigma^{a} L_{L}\right)\left(\bar{Q}_{L} \gamma_{\mu} \sigma^{a} Q_{L}\right), \\
& O_{\varphi l}^{(3)}=i\left(\varphi^{\dagger} D^{\mu} \sigma^{a} \varphi\right)\left(\bar{L}_{L} \gamma_{\mu} \sigma^{a} L_{L}\right)+\text { h.c. } \\
& O_{\varphi q}^{(3)}=i\left(\varphi^{\dagger} D^{\mu} \sigma^{a} \varphi\right)\left(\bar{Q}_{L} \gamma_{\mu} \sigma^{a} Q_{L}\right)+\text { h.c. }
\end{aligned}
$$

describing contact four-fermion interactions and gauge boson-fermion vertex corrections. Defining $\hat{\alpha}_{i}^{(3)}=\eta_{i} v^{2} / \Lambda_{i}^{2}$ (with $\eta_{i}= \pm 1$ ), one has

$$
\Delta_{\mathrm{CKM}}=4\left(\hat{\alpha}_{l l}^{(3)}-\hat{\alpha}_{l q}^{(3)}-\hat{\alpha}_{\varphi l}^{(3)}+\hat{\alpha}_{\varphi q}^{(3)}\right) .
$$

In specific SM extensions, the $\hat{\alpha}_{i}$ are functions of the underlying parameters. Therefore, via the above relation one can obtain the constraints from quark-lepton universality tests on any weakly-coupled SM extension.

Each of the $\hat{\alpha}$ coefficients also contributes to other lowand high-energy precision electroweak observables 82 . Therefore, we can now address in a model-independent way concrete questions such as the following:

- What is the maximal deviation $\left|\Delta_{\mathrm{CKM}}\right|$ allowed once all the precision electroweak constraints have been taken into account?

- Which observables provide the strongest constraints on the operators contributing to $\Delta_{\mathrm{CKM}}$ ?

In order to quantify the significance of the experimental constraints on CKM unitarity, we first calculate the range of $\Delta_{\mathrm{CKM}}\left(\hat{\alpha}_{i}\right)$ allowed by existing bounds from all precision electroweak measurements 76 . In terms of the best-fit values and the covariance matrix of the $\hat{\alpha}_{i} 82$ obtained from the fit to electroweak precision data, at $90 \%$ C.L. one has

$$
-9.5 \times 10^{-3} \leq \Delta_{\mathrm{CKM}} \leq 0.1 \times 10^{-3} .
$$

This result implies that a deviation from CKM unitarity at the level of $-1 \%$ is not ruled out by precision electroweak tests. A closer scrutiny of the precision data shows that the blame for large deviations of $\Delta_{\mathrm{CKM}}$ from zero could be attributed almost entirely to the operator $O_{l q}^{(3)}$, which is constrained relatively poorly from LEP2 hadronic cross section data, while the other three operators are severely constrained: $O_{l l}^{(3)}$ by the Fermi constant, $O_{\varphi q}^{(3)}$ by hadronic $Z$ decays, and $O_{\varphi l}^{(3)}$ by leptonic $Z$ decays.

The above discussion implies that even a percent-level test of CKM unitarity would provide information not available from other precision tests at low and high energies. Indeed, by Eq. (47), a test of CKM unitarity to better than one part in $10^{3}$ (e.g., with a $0.5 \%$ determination of $\left|V_{u s}\right|$ from kaon decays, combined with the $0.02 \%$ determination of $\left|V_{u d}\right|$ from nuclear beta decays) would probe new-physics effective scales $\Lambda$ on the order of $10 \mathrm{TeV}$. As we will show in Sect. 4, the current level of theoretical and experimental precision in the determination of $\left|V_{u s}\right|$ allows this prospect to be realized.

\subsubsection{Beyond $\mathrm{U}(3)^{5}$}

Corrections to the $\mathrm{U}(3)^{5}$ limit can be introduced both within MFV and via generic flavor structures. In chargedcurrent processes, the main effect of $\mathrm{U}(3)^{5}$ breaking is to turn on the chirality flipping (pseudo)scalar and tensor structures in Eq. (41). In MFV, the coefficients parameterizing deviations from $\mathrm{U}(3)^{5}$ are highly suppressed (the chirality flip is associated with insertions of Yukawa matrices). However, such suppression can be compensated by a corresponding $\tan \beta$ enhancement in models with two Higgs doublets of Type II, such as the Higgs sector of the minimal supersymmetric extension of the SM (MSSM)5 In this case, the leading non-standard contribution involves charged Higgs exchange. To one loop, this generates the coefficient

$$
\left[s_{R}\right]_{u s}=-\frac{\tan ^{2} \beta}{\left(1+\epsilon_{0} \tan \beta\right)} \frac{m_{\ell} m_{s}}{m_{H^{+}}^{2}},
$$

where $\epsilon_{0}$ is a correction factor which is negligible in the non-supersymmetric case, while it is $\mathcal{O}\left(1 / 16 \pi^{2}\right)$ in the MSSM [83] (see also [84, 85]). In $K_{\ell 2}$ decay, the $H^{ \pm}$ exchange amplitude destructively interferes with the SM $W^{ \pm}$amplitude; for large values of $\tan \beta(\sim 50)$ and low values of $m_{H^{ \pm}}(\sim 100 \mathrm{GeV})$, the $K_{\ell 2}$ rate can be decreased by as much as $5 \%$.

A highly sensitive probe of $\mathrm{U}(3)^{5}$ violating structures is therefore provided by comparing the value of $\left|V_{u s}\right|$ determined using $K_{\mu 2}$ decays, which are helicity suppressed, and $K_{\ell 3}$ decays, which are helicity allowed 6 In practice, to minimize the impact of the uncertainties from $f_{K}$ and the electromagnetic corrections for $K_{\mu 2}$, it is more convenient to consider the ratio

$$
R_{\mu 23}=\left(\frac{f_{K} / f_{\pi}}{f_{+}(0)}\right)^{-1}\left(\left|\frac{V_{u s}}{V_{u d}}\right| \frac{f_{K}}{f_{\pi}}\right)_{\mu 2} \frac{\left|V_{u d}\right|_{0^{+} \rightarrow 0^{+}}}{\left[\left|V_{u s}\right| f_{+}(0)\right]_{\ell 3}},
$$

which makes explicit contact with the results of Sects. 4.1 and 4.4. The hadronic uncertainties enter through the combination $\left(f_{K} / f_{\pi}\right) / f_{+}(0)$ and could be reduced if this quantity were to be directly computed on the lattice. Within the SM $R_{\mu 23}=R_{\ell 23}=1$, while the inclusion of Higgs-mediated scalar currents leads to

$$
R_{\mu 23} \approx\left|1-\frac{m_{K^{+}}^{2}}{m_{H^{+}}^{2}} \frac{\tan ^{2} \beta}{1+\epsilon_{0} \tan \beta}\right| .
$$

\footnotetext{
${ }^{5}$ In such models, $\tan \beta=v_{2} / v_{1}$ is the ratio of the two Higgs vacuum expectation values.

${ }^{6}$ In principle, the $H^{ \pm}$exchange amplitude affects $K_{e 3}$ and $K_{\mu 3}$ decays differently. For $K_{e 3}$, the effect is totally negligible, while for $K_{\mu 3}$, it is substantially smaller than for $K_{\mu 2}$, but not totally negligible. However, this effect is well below the present theoretical and experimental errors.
} 
Note that $R_{\mu 23}$ also provides interesting constraints on the existence of charged right-handed currents that appear at NLO in a not-quite-decoupling Higgsless effective theory 71, 86.

\section{Experimental inputs}

\subsection{Notes on fits to branching-ratio and lifetime measurements}

We perform separate fits to world data on the BRs and lifetimes for the $K_{L}, K_{S}$ and $K^{ \pm}$. The inputs to our fits are the observables actually measured by each single experiment, such as absolute BRs, ratios of BRs, lifetimes, or partial widths. For uncorrelated measurements with statistical and systematic errors quoted separately, we add the errors in quadrature. In many cases, the results for different quantities measured by the same experiment have correlated errors. The errors are then described by the covariance matrix, which must be provided by the experiment. The free parameters in our fits are the dominant BRs and the lifetime. In each case, the BRs are constrained to sum to unity. The penalty method [4] is used to implement this constraint. Once a first fit has been performed, scale factors are calculated and used as described in the general introduction to the Particle Data Group (PDG) compilation [87. The present versions of our fits make use of only published measurements. Moreover, for a measurement to be included in one of our fits, we require the following information to be available:

- for BR measurements, an explicit discussion of a satisfactory treatment of radiative corrections, especially when they are of comparable size to the experimental uncertainty;

- some details about the estimation of the systematic uncertainties;

- for correlated measurements from the same experiment, the complete covariance matrix.

\subsection{Dominant $K_{L}$ branching ratios and $\tau_{K_{L}}$}

Numerous measurements of the principal $K_{L}$ BRs, or of various ratios of these BRs, have been published in recent years.

The $\mathrm{KTeV}$ experiment has measured five ratios of the partial widths for the six main $K_{L}$ decays from independent samples of $10^{5}-10^{6}$ events collected with a single trigger 91. The $\mathrm{KTeV}$ results for the ratios $\operatorname{BR}\left(K_{\mu 3}\right) / \mathrm{BR}\left(K_{e 3}\right), \quad \operatorname{BR}\left(\pi^{+} \pi^{-} \pi^{0}\right) / \mathrm{BR}\left(K_{e 3}\right)$, $\mathrm{BR}\left(3 \pi^{0}\right) / \mathrm{BR}\left(K_{e 3}\right), \quad \operatorname{BR}\left(\pi^{+} \pi^{-}\right) / \mathrm{BR}\left(K_{e 3}\right), \quad$ and $\operatorname{BR}\left(2 \pi^{0}\right) / \operatorname{BR}\left(3 \pi^{0}\right)$, with total uncertainties ranging from 0.4 to $1.1 \%$, are listed in Table 2. The six decay modes involved account for more than $99.9 \%$ of the $K_{L}$ width, so $\mathrm{KTeV}$ combines the ratios to determine the absolute BRs. We instead use the five measured ratios in our global fit to $K_{L}$ BRs and lifetime. The correlations between the errors are provided by the experiment, and are taken into account in our fit.
NA48 has measured the ratio of the $K_{e 3}$ branching ratio to that for all $K_{L}$ decays to final states with two tracks 94. Using a sample of $8 \times 10^{7}$ events, they obtain $\mathrm{BR}\left(K_{e 3}\right) / \mathrm{BR}(2$ tracks $)=0.4978(35)$.

Using a sample of $13 \times 10^{6} \phi \rightarrow K_{L} K_{S}$ decays in which the $K_{S}$ decays to $\pi^{+} \pi^{-}$, providing normalization, KLOE has directly measured the BRs for the four main $K_{L}$ decay channels 90 . The results depend on the $K_{L}$ lifetime through the geometrical acceptance of the apparatus. The values listed in Table 2 were obtained using $\tau_{K_{L}}^{(0)}=51.54 \mathrm{~ns}$ as a reference value, and depend on the actual value of the lifetime as $d \mathrm{BR} / \mathrm{BR}=0.67 d \tau_{K_{L}} / \tau_{K_{L}}$. KLOE also reports results obtained using a constraint on the sum of the four measured BRs to solve for $\tau_{K_{L}}$, which significantly reduces the uncertainties on the BR measurements. In our $K_{L}$ fit, we use the values listed in Table 2 and explicitly include the lifetime dependence and other experimental correlations; we do not make use of the value of $\tau_{K_{L}}$ obtained in 90 .

KLOE has also measured $\tau_{K_{L}}$ directly, by fitting the proper decay time distribution using $K_{L} \rightarrow 3 \pi^{0}$ events, for which the reconstruction efficiency is high and uniform over a fiducial volume of $\sim 0.4 \beta \gamma c \tau_{K_{L}}$. They obtain $\tau_{K_{L}}=$ $50.92(30) \mathrm{ns}$ 88. We use this value in the fit.

There are two recent measurements of $\operatorname{BR}\left(\pi^{+} \pi^{-}\right) / \operatorname{BR}\left(K_{\ell 3}\right)$, in addition to the $\mathrm{KTeV}$ measurement of $\operatorname{BR}\left(\pi^{+} \pi^{-}\right) / \mathrm{BR}\left(K_{e 3}\right)$ discussed above. The KLOE collaboration obtains $\operatorname{BR}\left(\pi^{+} \pi^{-}\right) / \operatorname{BR}\left(K_{\mu 3}\right)=$ $7.275(68) \times 10^{-3} \quad 93$, while NA48 obtains $\mathrm{BR}\left(\pi^{+} \pi^{-}\right) / \mathrm{BR}\left(K_{e 3}\right)=4.826(27) \times 10^{-3}[92$. All of these measurements are fully inclusive of inner bremsstrahlung. The KLOE measurement is fully inclusive of the directemission (DE) component, DE contributes negligibly to the $\mathrm{KTeV}$ measurement, and a residual DE contribution of $0.19 \%$ has been subtracted from the NA48 value to obtain the number quoted above.

We fit the 12 recent measurements listed above, together with nine additional ratios of the BRs for subdominant decays. The complete list of 21 inputs is given in Table 2. As free parameters, our fit has the seven largest $K_{L}$ BRs (those to $K_{e 3}, K_{\mu 3}, 3 \pi^{0}, \pi^{+} \pi^{-} \pi^{0}, \pi^{+} \pi^{-}, \pi^{0} \pi^{0}$ and $\gamma \gamma$ ) and the $K_{L}$ lifetime. Our definition of $\operatorname{BR}\left(\pi^{+} \pi^{-}\right)$ is now fully inclusive of inner bremsstrahlung (IB), but exclusive of the DE component. The fit also includes two additional parameters necessary for the treatment of the DE component in the radiation-inclusive $\pi^{+} \pi^{-}$decay width: $\operatorname{BR}\left(\pi^{+} \pi^{-} \gamma\right)$ and $\operatorname{BR}\left(\pi^{+} \pi^{-} \gamma_{\mathrm{DE}}\right)$, the branching ratios for decays to states with a photon with decay-frame energy $E_{\gamma}^{*}>20 \mathrm{MeV}$, and with a photon from DE with $E_{\gamma}^{*}>$ $20 \mathrm{MeV}$, respectively. Other parameterizations are possible, but this one most closely represents the input data set and conforms to recent PDG usage. With 21 input measurements, 10 free parameters, and the constraint that the sum of the BRs (except for $\operatorname{BR}\left(\pi^{+} \pi^{-} \gamma\right)$, which is entirely included in the sum of $\operatorname{BR}\left(\pi^{+} \pi^{-}\right)$and $\left.\operatorname{BR}\left(\pi^{+} \pi^{-} \gamma_{\mathrm{DE}}\right)\right)$ equal unity, we have 12 degrees of freedom. The fit results are summarized in Table 3 . The fit gives $\chi^{2} /$ ndf $=19.8 / 12$ $(P=7.1 \%)$. The normalized residuals with respect to the result of the fit (the pulls) for each input are listed in Ta- 


\begin{tabular}{|c|c|c|c|c|}
\hline Parameter & Value & Source & Ref. & Pull \\
\hline$\tau_{K_{L}}$ & $50.92(30) \mathrm{ns}$ & KLOE & 88 & -0.8 \\
\hline$\tau_{K_{L}}$ & $51.54(44) \mathrm{ns}$ & Vosburgh & 89 & +0.9 \\
\hline $\operatorname{BR}\left(K_{e 3}\right)$ & $0.4049(21)$ & KLOE & 90 & -1.3 \\
\hline $\mathrm{BR}\left(K_{\mu 3}\right)$ & $0.2726(16)$ & KLOE & 90 & +0.5 \\
\hline $\operatorname{BR}\left(K_{\mu 3}\right) / \operatorname{BR}\left(K_{e 3}\right)$ & $0.6640(26)$ & $\mathrm{KTeV}$ & 91 & -1.1 \\
\hline $\operatorname{BR}\left(3 \pi^{0}\right)$ & $0.2018(24)$ & KLOE & 90 & +2.4 \\
\hline $\operatorname{BR}\left(3 \pi^{0}\right) / \operatorname{BR}\left(K_{e 3}\right)$ & $0.4782(55)$ & $\mathrm{KTeV}$ & 91 & -0.5 \\
\hline $\operatorname{BR}\left(\pi^{+} \pi^{-} \pi^{0}\right)$ & $0.1276(15)$ & KLOE & 90 & +1.0 \\
\hline $\operatorname{BR}\left(\pi^{+} \pi^{-} \pi^{0}\right) / \operatorname{BR}\left(K_{e 3}\right)$ & $0.3078(18)$ & $\mathrm{KTeV}$ & 91 & -0.8 \\
\hline $\operatorname{BR}\left(\pi^{+} \pi^{-}\right) / \operatorname{BR}\left(K_{e 3}\right)$ & $0.004856(29)$ & $\mathrm{KTeV}$ & 91 & +0.3 \\
\hline $\operatorname{BR}\left(\pi^{+} \pi^{-}\right) / \operatorname{BR}\left(K_{e 3}\right)$ & $0.004826(27)$ & NA48 & 92 & -0.8 \\
\hline $\operatorname{BR}\left(\pi^{+} \pi^{-}\right) / \operatorname{BR}\left(K_{\mu 3}\right)$ & $0.007275(68)$ & KLOE & 93 & -1.5 \\
\hline $\mathrm{BR}\left(K_{e 3}\right) / \mathrm{BR}(2$ tracks $)$ & $0.4978(35)$ & NA48 & 94 & -0.8 \\
\hline $\operatorname{BR}\left(\pi^{0} \pi^{0}\right) / \operatorname{BR}\left(3 \pi^{0}\right)$ & $0.004446(25)$ & $\mathrm{KTeV}$ & 91 & +0.6 \\
\hline $\operatorname{BR}\left(\pi^{0} \pi^{0}\right) / \operatorname{BR}\left(\pi^{+} \pi^{-}\right)$ & $0.4391(13)$ & PDG ETAFIT & 87 & -0.5 \\
\hline $\operatorname{BR}(\gamma \gamma) / \operatorname{BR}\left(3 \pi^{0}\right)$ & $0.00279(3)$ & KLOE & 95 & -0.5 \\
\hline $\operatorname{BR}(\gamma \gamma) / \operatorname{BR}\left(3 \pi^{0}\right)$ & $0.00281(2)$ & NA48 & 96 & +0.3 \\
\hline $\operatorname{BR}\left(\pi^{+} \pi^{-} \gamma\right) / \operatorname{BR}\left(\pi^{+} \pi^{-}\right)$ & $0.0208(3)$ & $\mathrm{KTeV}$ & 97 & 0.0 \\
\hline $\operatorname{BR}\left(\pi^{+} \pi^{-} \gamma_{\mathrm{DE}}\right) / \mathrm{BR}\left(\pi^{+} \pi^{-} \gamma\right)$ & $0.689(21)$ & $\mathrm{KTeV}$ & 98 & +0.2 \\
\hline $\operatorname{BR}\left(\pi^{+} \pi^{-} \gamma_{\mathrm{DE}}\right) / \operatorname{BR}\left(\pi^{+} \pi^{-} \gamma\right)$ & $0.683(11)$ & $\mathrm{KTeV}$ & 97 & -0.1 \\
\hline $\operatorname{BR}\left(\pi^{+} \pi^{-} \gamma_{\mathrm{DE}}\right) / \operatorname{BR}\left(\pi^{+} \pi^{-} \gamma\right)$ & $0.685(41)$ & E731 & 99 & 0.0 \\
\hline
\end{tabular}

Table 2. Input data used for the fit to $K_{L}$ BRs and lifetime. For each measurement, the normalized residual with respect to the results of the fit is listed in the last column.

\begin{tabular}{|c|c|c|c|c|c|c|c|c|c|c|c|}
\hline Parameter & $\overline{\overline{\text { Value }}}$ & $\overline{\bar{S}}$ & \multicolumn{9}{|c|}{ Correlation matrix (\%) } \\
\hline $\operatorname{BR}\left(K_{e 3}\right)$ & $0.4056(9)$ & 1.3 & -29 & -45 & -30 & +6 & +10 & -27 & -27 & +8 & +15 \\
\hline $\mathrm{BR}\left(K_{\mu 3}\right)$ & $0.2704(10)$ & 1.5 & & -50 & 0 & -3 & -10 & -32 & -35 & +13 & -16 \\
\hline $\operatorname{BR}\left(3 \pi^{0}\right)$ & $0.1952(9)$ & 1.2 & & & -37 & -1 & +9 & +56 & +63 & -13 & +12 \\
\hline $\operatorname{BR}\left(\pi^{+} \pi^{-} \pi^{0}\right)$ & $0.1254(6)$ & 1.3 & & & & -4 & -16 & -15 & -21 & -5 & -20 \\
\hline $\mathrm{BR}\left(\pi^{+} \pi^{-}\right)$ & $1.967(7) \times 10^{-3}$ & 1.1 & & & & & +14 & +34 & +1 & -3 & +19 \\
\hline $\operatorname{BR}\left(\pi^{+} \pi^{-} \gamma\right)$ & $4.15(9) \times 10^{-5}$ & 1.6 & & & & & & +16 & +8 & -3 & +74 \\
\hline $\mathrm{BR}\left(\pi^{+} \pi^{-} \gamma_{\mathrm{DE}}\right)$ & $2.84(8) \times 10^{-5}$ & 1.3 & & & & & & & +35 & -10 & +22 \\
\hline $\operatorname{BR}\left(2 \pi^{0}\right)$ & $8.65(4) \times 10^{-4}$ & 1.4 & & & & & & & & -8 & +10 \\
\hline $\operatorname{BR}(\gamma \gamma)$ & $5.47(4) \times 10^{-4}$ & 1.1 & & & & & & & & & -4 \\
\hline$\tau_{K_{L}}$ & $51.16(21) \mathrm{ns}$ & 1.1 & & & & & & & & & \\
\hline
\end{tabular}

Table 3. Results of fit to $K_{L}$ BRs and lifetime.

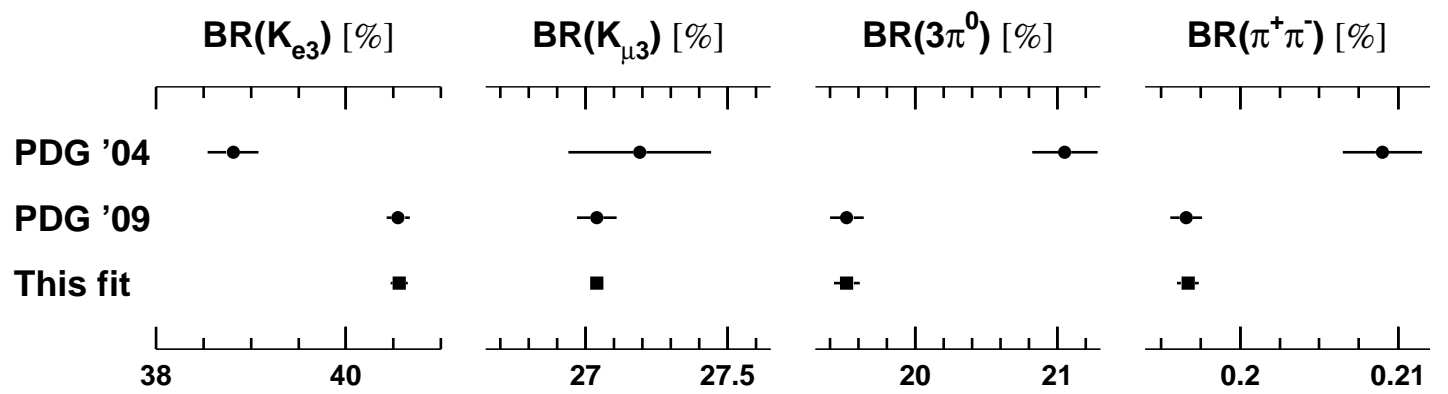

Fig. 3. Evolution of average values for main $K_{L}$ BRs.

ble 2. The evolution of the average values of the BRs for $K_{\ell 3}$ decays and for the important normalization channels is shown in Fig. 3 .

As seen from Fig. 3. both our fit and the recent PDG fit 87. differ substantially in their results from the 2004 PDG fit 100. This is due to the addition to the world data set after 2004 of most of the recent data discussed above, and to the subsequent elimination of numerous old measurements - many with questionable radiative corrections and/or unreported correlations - used in previous fits. Between 2004 and today, the world-average BRs for the $K_{e 3}, 3 \pi^{0}$, and $\pi^{+} \pi^{-}$decays have shifted by $+6 \sigma$, $-6 \sigma$, and $-5 \sigma$, respectively, leading to the following consequences: 
- The world-average value for the ratio $\Gamma\left(K_{\mu 3}\right) / \Gamma\left(K_{e 3}\right)$ changes from $0.701(8)$ to $0.6668(28)$, procuring better agreement with the value expected from lepton universality, as discussed in Sect. 4.3.

- The world-average value for the amplitude ratio $\left|\eta_{+-}\right|$, a parameter of $C P$ violation in the $K_{S} K_{L}$ system, changes from $2.286(18) \times 10^{-3}$ to $2.231(10) \times 10^{-3}$, $\mathrm{a}-2.7 \sigma$ difference. (For this comparison we are interested mainly in the effect of the new $K_{L}$ data, so we use the results of the fit to $K_{S}$ rate data in Table 4 to evaluate $\left|\eta_{+-}\right|$for both years.)

Figure 3 also shows that differences between the results of our fit and the most recent PDG fit are minor. These fits differ principally in the following respects:

- The PDG fit uses five of the six $\mathrm{KTeV}$ values for the dominant $K_{L}$ BRs, while our fit uses the five ratios directly.

- The PDG fit uses three of the four KLOE values for the dominant $K_{L}$ BRs obtained after imposing the constraint on the sum, as well as the KLOE value of $\tau_{K_{L}}$ obtained by imposing this constraint. We use the original KLOE values with their lifetime dependence and omit the value of $\tau_{K_{L}}$ obtained from the constraint on the sum.

- The PDG fit includes $\mathrm{BR}\left(e^{+} e^{-} \gamma\right)$ as a free parameter, and thus makes use of four measurements not used in our fit.

Our treatment of the contribution from DE in the $\pi^{+} \pi^{-} \gamma$ decay is the same as that used by the PDG, but we omit the measurement of $\operatorname{BR}\left(\pi^{+} \pi^{-} \gamma\right) / \operatorname{BR}\left(\pi^{+} \pi^{-}\right)$from $\operatorname{E} 731$ [99, because while the photon energy cutoff in the numerator is well defined $\left(E_{\gamma}^{*}>20 \mathrm{MeV}\right)$, the requirement used in this measurement for events in the normalization channel to contain no calorimeter clusters other than those from the $\pi^{+} \pi^{-}$leads to difficulties of interpretation.

\subsection{Dominant $K_{S}$ branching ratios and $\tau_{K_{S}}$}

The KLOE collaboration has measured the ratio $\operatorname{BR}\left(K_{S} \rightarrow \pi e \nu\right) / \operatorname{BR}\left(K_{S} \rightarrow \pi^{+} \pi^{-}\right)=10.19(13) \times 10^{-3}$ with $1.3 \%$ precision [101, making possible an independent determination of $\left|V_{u s}\right| f_{+}(0)$ to better than $0.7 \%$. In [102, they combine the above measurement with their measurement $\operatorname{BR}\left(K_{S} \rightarrow \pi^{+} \pi^{-}\right) / \operatorname{BR}\left(K_{S} \rightarrow \pi^{0} \pi^{0}\right)=2.2459(54)$. Using the constraint that the $K_{S}$ BRs must sum to unity and assuming the universality of lepton couplings, they determine the BRs for the $\pi^{+} \pi^{-}, \pi^{0} \pi^{0}, K_{e 3}$, and $K_{\mu 3}$ decays.

Our fit is an extension of the analysis in 102 . We perform a fit to the data on the $K_{S}$ BRs to $\pi^{+} \pi^{-}, \pi^{0} \pi^{0}$, and $K_{e 3}$ that uses, in addition to the above two measurements:

- the measurement from NA48, $\Gamma\left(K_{S} \rightarrow \pi e \nu\right) / \Gamma\left(K_{L} \rightarrow\right.$ $\pi e \nu)=0.993(34)$ 103, where the denominator is obtained from the results of our $K_{L}$ fit;

- the measurements from NA48, $\tau_{K_{S}}=89.598(70) \mathrm{ps}$ [104], and $\mathrm{KTeV}, \tau_{K_{S}}=89.58(13)$ ps [105], both obtained without the assumption of $C P T$ symmetry;

\begin{tabular}{lc|rrrr}
\hline \hline Parameter & Value & \multicolumn{3}{|c}{ Correlation matrix (\%) } \\
\hline $\operatorname{BR}\left(\pi^{+} \pi^{-}\right)$ & $0.6920(5)$ & -100 & +4 & +3 & +0 \\
$\operatorname{BR}\left(\pi^{0} \pi^{0}\right)$ & $0.3069(5)$ & & -6 & -6 & +0 \\
$\operatorname{BR}\left(K_{e 3}\right)$ & $7.05(8) \times 10^{-4}$ & & & +98 & +1 \\
$\operatorname{BR}\left(K_{\mu 3}\right)$ & $4.69(6) \times 10^{-4}$ & & & & +1 \\
$\tau_{K_{S}}$ & $89.59(6) \mathrm{ps}$ & & & & \\
\hline \hline
\end{tabular}

Table 4. Results of fit to $K_{S}$ BRs and lifetime.

- the result $\mathrm{BR}\left(K_{\mu 3}\right) / \mathrm{BR}\left(K_{e 3}\right)=0.6655(15)$ obtained from the assumption of universal lepton couplings, the values of $\Lambda_{+}$and $\ln C$, the parameters of the dispersive representation of the vector and scalar form factors, obtained from our fit to form factor data discussed in Sect. 3.5.2 and the long-distance electromagnetic corrections discussed in Sect. 2.2

The free parameters are the four BRs listed above plus $\tau_{K_{S}}$. With six inputs and one constraint (on the sum of the BRs), the fit has one degree of freedom and gives $\chi^{2} / \mathrm{ndf}=$ $0.015 / 1(P=90 \%)$. The scale factor $S$ is not different from unity for any of the output values. The results of the fit are listed in Table 4

\subsection{Dominant $K^{ \pm}$branching ratios and $\tau_{K^{ \pm}}$}

Several recent measurements contribute significant new information on the rates for the dominant $K^{ \pm}$decays. In addition, we have recently carried out a comprehensive, critical survey of the $K^{ \pm}$data set, which led to the elimination of numerous older measurements previously used in the fit. The input data used in our current fit to determine the dominant $K^{ \pm}$BRs and lifetime are summarized in Table 5 ,

The 2003 measurement of $\mathrm{BR}\left(K_{e 3}^{+}\right)$by E865 116 was the first of the recent-generation measurements of semileptonic kaon BRs, and gave a value for $\left|V_{u s}\right|$ consistent with unitarity. The quantity actually measured was $\mathrm{BR}\left(K_{e 3}^{+}\right) /\left(\mathrm{BR}\left(\pi^{+} \pi^{0}\right)+\mathrm{BR}\left(K_{\mu 3}^{+}\right)+\mathrm{BR}\left(\pi^{+} \pi^{0} \pi^{0}\right)\right)$, where one $\pi^{0}$ in the final state was required to undergo Dalitz decay. (Throughout the remainder of this section, we use $\pi$ to denote the charged pion when no confusion results.)

In 2007, the NA48/2 collaboration published measurements of the ratios $\operatorname{BR}\left(K_{e 3}\right) / \mathrm{BR}\left(\pi \pi^{0}\right)$ and $\operatorname{BR}\left(K_{\mu 3}\right) / \operatorname{BR}\left(\pi \pi^{0}\right)$ obtained with simultaneous $K^{+}$and $K^{-}$beams 117,121. For each type of $K_{\ell 3}$ decay (i.e., to $e$ and $\mu$ ), about $50 \mathrm{k} K^{+}$and $30 \mathrm{k} K^{-}$decays were collected. The results for these ratios in Table 5 are correlated with $\rho=+0.19[122$. The dominant uncertainties are from sample statistics.

ISTRA+ has also performed a measurement of $\operatorname{BR}\left(K_{e 3}^{-}\right) / \operatorname{BR}\left(\pi \pi^{0}\right)$ with $0.6 \%$ precision 123 . The result, however, has not been officially published and is therefore not used in our fit.

KLOE has measured the absolute BRs for the $K_{e 3}$ and $K_{\mu 3}$ decays [115. In $\phi \rightarrow K^{+} K^{-}$events, $K^{+}$decays into $\mu^{+} \nu$ or $\pi^{+} \pi^{0}$ are used to tag a $K^{-}$beam, and vice versa. KLOE performs four separate measurements for each $K_{\ell 3}$ $\mathrm{BR}$, corresponding to the different combinations of kaon 


\begin{tabular}{|c|c|c|c|c|}
\hline Parameter & Value & Source & Ref. & Pull \\
\hline$\tau_{K^{ \pm}}$ & $12.422(40) \mathrm{ns}$ & Koptev* & 106 & +0.9 \\
\hline$\tau_{K^{ \pm}}$ & $12.380(16) \mathrm{ns}$ & Ott & 107 & -0.3 \\
\hline$\tau_{K^{ \pm}}$ & $12.443(38) \mathrm{ns}$ & Fitch & 108 & +1.5 \\
\hline$\tau_{K^{ \pm}}$ & $12.347(30) \mathrm{ns}$ & KLOE & 109 & -1.2 \\
\hline $\operatorname{BR}\left(K_{\mu 2}\right)$ & $0.6366(17)$ & KLOE & 110 & +1.1 \\
\hline $\operatorname{BR}\left(\pi \pi^{0}\right)$ & $0.2065(9)$ & KLOE & 111 & +0.5 \\
\hline $\operatorname{BR}\left(\pi \pi^{0}\right) / \operatorname{BR}\left(K_{\mu 2}\right)$ & $0.3329(48)$ & PS183 & 112 & +1.7 \\
\hline $\operatorname{BR}\left(\pi \pi^{0}\right) / \operatorname{BR}\left(K_{\mu 2}\right)$ & $0.3355(57)$ & Weissenberg & 113 & +1.9 \\
\hline $\operatorname{BR}\left(\pi \pi^{0}\right) / \operatorname{BR}\left(K_{\mu 2}\right)$ & $0.3277(65)$ & Auerbach & 114 & +0.5 \\
\hline $\operatorname{BR}\left(K_{e 3}\right)$ & $0.04965(53)$ & KLOE & 115 & -2.1 \\
\hline $\operatorname{BR}\left(K_{e 3}\right) / \operatorname{BR}\left(\pi \pi^{0}+K_{\mu 3}+\pi \pi^{0} \pi^{0}\right)$ & $0.1962(36)$ & BNL-E865 & 116 & -0.3 \\
\hline $\operatorname{BR}\left(K_{e 3}\right) / \operatorname{BR}\left(\pi \pi^{0}\right)$ & $0.2470(10)$ & $\mathrm{NA} 48 / 2$ & 117 & +0.6 \\
\hline $\operatorname{BR}\left(K_{\mu 3}\right)$ & $0.03233(39)$ & KLOE & 115 & -3.2 \\
\hline $\operatorname{BR}\left(K_{\mu 3}\right) / \operatorname{BR}\left(\pi \pi^{0}\right)$ & $0.1637(7)$ & $\mathrm{NA} 48 / 2$ & 117 & +1.0 \\
\hline $\operatorname{BR}\left(K_{\mu 3}\right) / \operatorname{BR}\left(K_{e 3}\right)$ & $0.671(11)$ & KEK-E246 & 118 & +0.9 \\
\hline $\operatorname{BR}\left(\pi \pi^{0} \pi^{0}\right)$ & $0.01763(26)$ & KLOE & 119 & +0.2 \\
\hline $\operatorname{BR}\left(\pi \pi^{0} \pi^{0}\right) / \operatorname{BR}(\pi \pi \pi)$ & $0.303(9)$ & Bisi & 120 & -0.4 \\
\hline
\end{tabular}

Table 5. Input data used for the fit to $K^{ \pm}$BRs and lifetime. For each measurement, the normalized residual with respect to the results of the fit is listed in the last column. The two 1995 values of $\tau_{K^{ \pm}}$from Koptev et al. are averaged with $S=1.6$ before being included in the fit as a single value.

charge and tagging decay. As in the case of KLOE's absolute BR measurements for the $K_{L}$, there is some dependence on the lifetime. The final values for $\operatorname{BR}\left(K_{e 3}\right)$ and $\operatorname{BR}\left(K_{\mu 3}\right)$ in Table 5 are referred to $\tau_{K^{ \pm}}=12.385 \mathrm{~ns}$ and depend on the actual value of the lifetime as $d \mathrm{BR} / \mathrm{BR}=$ $-0.45 d \tau_{K^{ \pm}} / \tau_{K^{ \pm}}$. The uncertainties are correlated, with $\rho=+0.63$.

As seen from Table 5, KLOE has also measured the absolute BRs for the important normalization channels $K^{+} \rightarrow \pi^{+} \pi^{0}$ [111] and $K^{+} \rightarrow \mu^{+} \nu$ [110], using $K^{-} \rightarrow$ $\mu^{-} \bar{\nu}$ decays to tag. Again, our fit takes into account the correlation between these values, as well as their dependence on the $K^{ \pm}$lifetime.

One of the primary motives for our critical survey of $K^{ \pm}$decay rate data was the poor consistency of the available lifetime measurements. The 2007 PDG average value for $\tau_{K^{ \pm}}, 12.385(25) \mathrm{ns}$, is nominally quite precise. However, the error is scaled by 2.1 , and the confidence level for the average is $0.17 \% 124$.

Our survey of the older measurements of $\tau_{K^{ \pm}}$led to the elimination of the 1967 result from Lobkowicz et al. [125], because the experiment was much more suited for measuring the difference between $\tau_{K^{+}}$and $\tau_{K^{-}}$than it was for the absolute measurement of either lifetime. The stopped- $K^{+}$ measurement described in the 1995 paper from Koptev et al. 106 makes use of the surface-muon technique, in which kaons are produced and stopped in the same target. The two results obtained using different target materials are in marginal agreement, with $S=1.6$. We have been able to identify neither a reason for the discrepancy, nor a reason for the exclusion of the measurement, and so we include in our fit the average of the two results, with the scale factor applied to the error to reflect their disagreement.

In 2008, KLOE published a new measurement of $\tau_{K^{ \pm}}$ 109. The new KLOE result in Table [5] is the average of separate measurements for $K_{\mu 2}$-tagged $K^{+}$and $K^{-}$ decays using two different techniques for each charge. In the first technique, the path length of the tagged kaon from production to decay was measured using an inclusive sample. In the second technique, the tagged kaon was required to decay to a final state containing a $\pi^{0}$, and the decay time was measured using the photon clusters in the calorimeter.

Our fit for the six largest $K^{ \pm}$branching ratios and lifetime uses the 17 measurements in Table 5. The fit has seven free parameters and one constraint, giving 11 degrees of freedom. We obtain the results in Table 6. The fit gives $\chi^{2} / \mathrm{ndf}=25.8 / 11(P=0.69 \%)$. The comparatively low $P$-value reflects some tension between the KLOE and NA48/2 measurements of the $K_{\ell 3}$ branching ratios.

For comparison, the most recent PDG fit 87 uses 32 measurements to determine seven BRs (including that for the $K_{e 4}$ decay to $\left.\pi^{0} \pi^{0} e^{ \pm} \nu\right)$ and the lifetime, and has $\chi^{2} /$ ndf $=52 / 25(P=0.13 \%)$. The main differences between our fit and the current PDG fit, besides the treatment of the $K^{ \pm}$lifetime data described above, lie in the elimination of ten older measurements of various BRs, including the five absolute BR measurements of Chiang et al. 126. In that study, absolute measurements of the six largest BRs were performed; the values were improved by constraining the sum of the six BRs to unity. Neither the original measurements nor the covariance matrix are reported. Moreover, radiative corrections appear not to have been applied when obtaining the $\mathrm{BR}$ values.

Both the significant evolution of the average values of the $K_{\ell 3}$ BRs over time and the remaining difference between our results and those of the PDG are evident in Fig. 4. 


\begin{tabular}{|c|c|c|c|c|c|c|c|c|}
\hline Parameter & Value & $S$ & \multicolumn{6}{|c|}{ Correlation matrix (\%) } \\
\hline $\operatorname{BR}\left(K_{\mu 2}\right)$ & $63.47(18) \%$ & 1.3 & -39 & -75 & -33 & -28 & -36 & +12 \\
\hline $\operatorname{BR}\left(\pi \pi^{0}\right)$ & $20.61(8) \%$ & 1.1 & & -26 & +61 & +38 & -13 & -11 \\
\hline $\mathrm{BR}(\pi \pi \pi)$ & $5.73(16) \%$ & 1.2 & & & -22 & -17 & +36 & -5 \\
\hline $\operatorname{BR}\left(K_{e 3}\right)$ & $5.078(31) \%$ & 1.3 & & & & +47 & -10 & -13 \\
\hline $\mathrm{BR}\left(K_{\mu 3}\right)$ & $3.359(32) \%$ & 1.9 & & & & & -8 & -4 \\
\hline $\operatorname{BR}\left(\pi \pi^{0} \pi^{0}\right)$ & $1.757(24) \%$ & 1.0 & & & & & & -1 \\
\hline$\tau_{K^{ \pm}}$ & $12.384(15) \mathrm{ns}$ & 1.2 & & & & & & \\
\hline
\end{tabular}

Table 6. Results of fit to $K^{ \pm}$BRs and lifetime.

$\operatorname{BR}\left(\mathbf{K}_{\mathrm{e} 3}\right)[\%]$

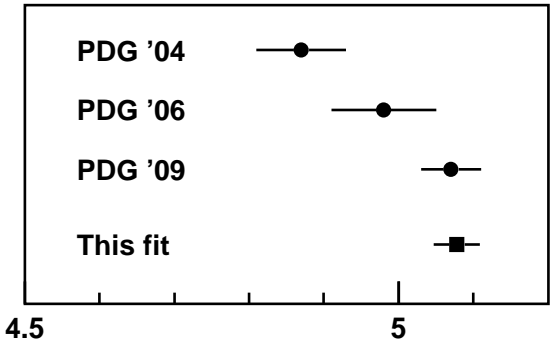

$\operatorname{BR}(\mu \nu)[\%]$

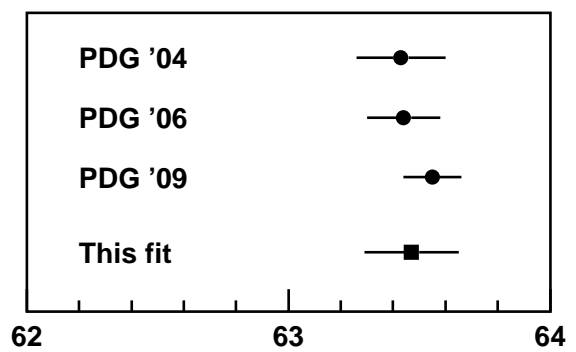

$\mathbf{B R}\left(\mathbf{K}_{\mu 3}\right)[\%]$

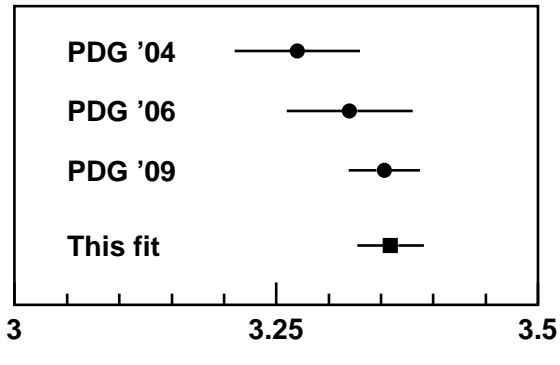

$\operatorname{BR}\left(\pi \pi^{0}\right)[\%]$

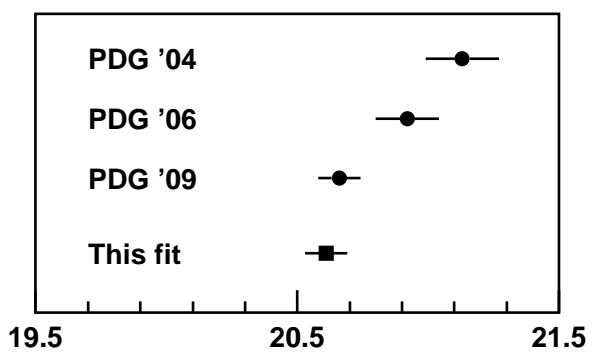

Fig. 4. Evolution of average values for main $K^{ \pm}$BRs.

\begin{tabular}{lccc}
\hline \hline Experiment & $\lambda_{+}^{\prime} \times 10^{3}$ & $\lambda_{+}^{\prime \prime} \times 10^{3}$ & $\rho\left(\lambda_{+}^{\prime}, \lambda_{+}^{\prime \prime}\right)$ \\
\hline KLOE & $25.5 \pm 1.8$ & $1.4 \pm 0.8$ & -0.95 \\
KTeV & $21.67 \pm 1.99$ & $2.87 \pm 0.78$ & -0.97 \\
NA48 & $28.0 \pm 2.4$ & $0.4 \pm 0.9$ & -0.88 \\
ISTRA + & $24.85 \pm 1.66$ & $1.92 \pm 0.94$ & -0.95 \\
\hline \hline
\end{tabular}

Table 7. Quadratic form factor parameters for $K_{e 3}$ decays. The values from NA48 and ISTRA+ have been converted for use with the notation of Eq. (28). The values of $\rho$ from NA48 and ISTRA+ were communicated privately [122,127.

\subsection{Measurements of $K_{\ell 3}$ form factor parameters}

\subsection{1 $K_{e 3}$ form factor parameters}

KLOE 128, KTeV [129, NA48 [130, and ISTRA+ 131] have all performed recent measurements of the quadratic parameters $\lambda_{+}^{\prime}$ and $\lambda_{+}^{\prime \prime}$ of the vector form factor for $K_{e 3}$ decays (see Eq. (28)). The data are listed in Table 7 and represented graphically in Fig. 5 .

Table 8 gives the results of a fit to the $K_{L}$ and $K^{-}$ data in the first column, and to the $K_{L}$ data only in the second column. With correlations taken into account, both fits give good values of $\chi^{2} / \mathrm{ndf}$. The significance of the quadratic term is $4.2 \sigma$ from the fit to all data, and $3.5 \sigma$

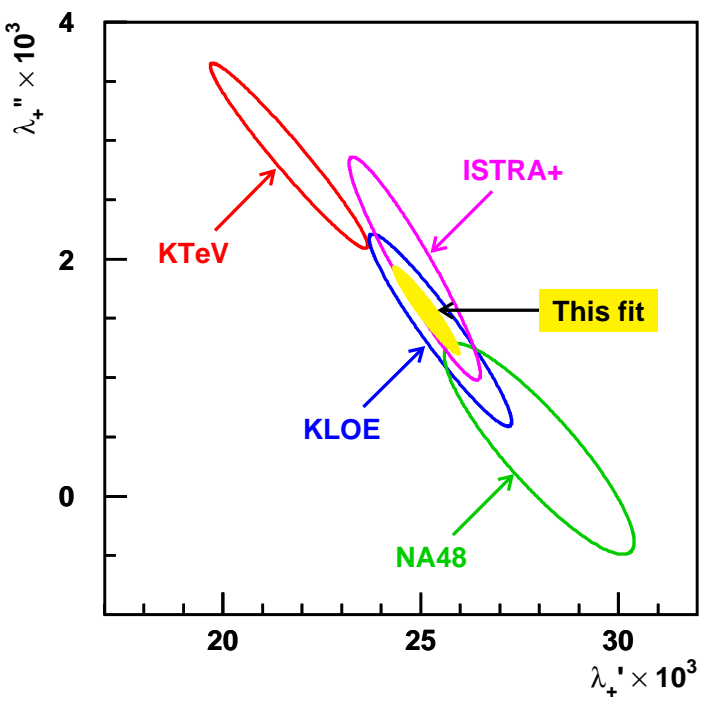

Fig. 5. Recent measurements of $K_{e 3}$ vector form factor parameters. The yellow ellipse shows the result of a fit to all data.

from the fit to $K_{L}$ data only. Including or excluding the $K^{-}$slopes has little impact on the values of $\lambda_{+}^{\prime}$ and $\lambda_{+}^{\prime \prime}$; in 


\begin{tabular}{lcc}
\hline \hline & $K_{L}$ and $K^{-}$data & $K_{L}$ data only \\
& 4 measurements & 3 measurements \\
& $\chi^{2} / \mathrm{ndf}=5.3 / 6(51 \%)$ & $\chi^{2} / \mathrm{ndf}=4.7 / 4(32 \%)$ \\
\hline$\lambda_{+}^{\prime} \times 10^{3}$ & $25.1 \pm 0.9$ & $24.9 \pm 1.1$ \\
$\lambda_{+}^{\prime \prime} \times 10^{3}$ & $1.6 \pm 0.4$ & $1.6 \pm 0.5$ \\
$\rho\left(\lambda_{+}^{\prime}, \lambda+^{\prime \prime}\right)$ & -0.94 & -0.95 \\
$I\left(K_{e 3}^{0}\right)$ & $0.15463(21)$ & $0.15454(29)$ \\
$I\left(K_{e 3}^{ \pm}\right)$ & $0.15900(22)$ & $0.15890(30)$ \\
\hline \hline
\end{tabular}

Table 8. Average of quadratic fit results for $K_{e 3}$ slopes.

\begin{tabular}{|c|c|c|c|c|}
\hline Experiment & 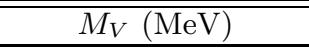 & Ref. & $\Lambda_{+} \times 10^{3}$ & Ref. \\
\hline KLOE & $870 \pm 6 \pm 7$ & 128 & $25.74 \pm 0.53$ & 128 \\
\hline $\mathrm{KTeV}$ & $881.03 \pm 7.11$ & 129 & $25.17 \pm 0.48$ & 68 \\
\hline NA48 & $859 \pm 18$ & 130 & $26.4 \pm 1.1$ & 130 \\
\hline ISTRA+ & $861.6 \pm 6.5 \pm 5.0$ & 127 & $26.20 \pm 0.56$ & 127 \\
\hline \multirow[t]{4}{*}{ Average } & $871 \pm 5$ & & $25.70 \pm 0.42$ & \\
\hline & $\chi^{2} / \mathrm{ndf}=3.8 / 3$ & & $\chi^{2} / \mathrm{ndf}=2.42 / 3$ & \\
\hline & $I\left(K_{e 3}^{0}\right)=0.15480(18)$ & & $I\left(K_{e 3}^{0}\right)=0.15478(18)$ & \\
\hline & $I\left(K_{e 3}^{ \pm}\right)=0.15918(19)$ & & $I\left(K_{e 3}^{ \pm}\right)=0.15924(19)$ & \\
\hline
\end{tabular}

Table 9. $K_{e 3}$ form factor parameters for the pole and dispersive parameterizations. Values of $\Lambda_{+}$from KLOE and NA48 were obtained from the pole fit results from those experiments.

particular, the values of the phase space integrals change by just $0.06 \%$. The errors on the phase space integrals are significantly smaller when the $K^{-}$data are included in the average. The results of the fit to all data are plotted as the yellow ellipse in Fig. 5.

All four experiments have fitted their $K_{e 3}$ data using the pole parameterization of Eq. (29), and obtain the values shown in the left part of Table 9 for the pole mass $M_{V}$. The average value is $M_{V}=871 \pm 5 \mathrm{MeV}$ with $\chi^{2} /$ ndf $=3.8 / 3(P=28.9 \%)$. The individual values are quite compatible with each other, and their average is reasonably close to the mass of the $K^{*}(892)$. The values of the integrals $I\left(K_{e 3}^{0}\right)$ and $I\left(K_{e 3}^{ \pm}\right)$as evaluated from the pole fit results are just $0.11 \%$ higher than the values obtained from the quadratic fit results.

The dispersive parameterization of the vector form factor of Eq. (38) is similar to the pole parameterization, but better motivated theoretically. KTeV and ISTRA+ have used this form to fit their $K_{e 3}$ data, while results for KLOE and NA48 can be obtained from the corresponding values of $M_{V}$ via $\Lambda_{+}=\left(m_{\pi^{ \pm}} / M_{V}\right)^{2}$. These results are listed in the right part of Table 9, together with our average. The uncertainties on the values from the individual experiments do not include the contribution arising from the representation of the form factor phase in the dispersive parameterization (see discussion in Ref. 68). This contribution is common to all experiments, and is included as an additional uncertainty of $0.30 \times 10^{-3}$ on our average value of $\Lambda_{+}$, which is propagated in the evaluation of the phase space integrals.

\subsection{2 $K_{\ell 3}$ form factor parameters}

KLOE, KTeV, NA48, and ISTRA+ have all performed measurements of the $K_{\mu 3}$ form factor parameters using the quadratic and linear parameterizations for the vector and scalar form factor parameters, respectively. The values of $\lambda_{+}^{\prime}$ and $\lambda_{+}^{\prime \prime}$ from $K_{e 3}$ decays may be averaged with the values of $\lambda_{+}^{\prime}, \lambda_{+}^{\prime \prime}$, and $\lambda_{0}$ from $K_{\mu 3}$ decays, with parameter correlations taken into account. KTeV and KLOE provide $K_{e 3}-K_{\mu 3}$ averages for the slope parameters; we calculate the averages for NA48 and ISTRA + . The $K_{e 3}-K_{\mu 3}$ average values for $\lambda_{+}^{\prime}, \lambda_{+}^{\prime \prime}$, and $\lambda_{0}$ from each experiment are listed in Table 10, the $1 \sigma$ confidence contours for each pair of parameters are plotted in Fig. 6. The only significant difference between the results presented in Table 10 and Fig. 6] and the corresponding presentation in Ref. 4 is that the final ISTRA+ systematics are now correctly included. As has been previously noted [4,135, the NA48 result is incompatible with all of the other measurements. Our average of the results from all four experiments is plotted in Fig. 6 as the cyan ellipse. This average gives $\chi^{2} /$ ndf $=48 / 9\left(P=3 \times 10^{-7}\right)$; the errors on $\lambda_{+}^{\prime}$ and $\lambda_{+}^{\prime \prime}$ are scaled by 1.4 in this case, while the error on $\lambda_{0}$ is scaled by 2.2 . If instead of the NA48 $K_{e 3}-K_{\mu 3}$ average from Table 10, only the $K_{e 3}$ measurement from NA48 [130] is used, much greater consistency is observed. The resulting average is plotted as the yellow ellipse in Fig. 6. this average gives $\chi^{2} / \mathrm{ndf}=12.1 / 8(P=14.5 \%)$.

Apart from concerns about the consistency of the data set, as described in Sect. 2.2.5, the use of a linear parameterization for the scalar form factor is inherently problematic; the use of a Class-I parameterization for the form factors such as the dispersive parameterization of Eqs. (33) and (37) is greatly preferred. Recently KLOE, KTeV, NA48, and ISTRA + have obtained results for the parameters $\left(\Lambda_{+}, \ln C\right)$ from fits to their $K_{\ell 3}$ data. These results are listed in Table 11 and shown graphically in Fig. 7. For each experiment, the results for $K_{e 3}$ and $K_{\mu 3}$ decays are averaged. As in Table 9, in Table 11 the uncertainties 


\begin{tabular}{lccccccr}
\hline \hline Experiment & $\lambda_{+}^{\prime} \times 10^{3}$ & $\lambda_{+}^{\prime \prime} \times 10^{3}$ & $\lambda_{0} \times 10^{3}$ & $\rho\left(\lambda_{+}^{\prime}, \lambda_{+}^{\prime \prime}\right)$ & $\rho\left(\lambda_{+}^{\prime}, \lambda_{0}\right)$ & $\rho\left(\lambda_{+}^{\prime \prime}, \lambda_{0}\right)$ & Refs. \\
\hline KTeV & $20.64 \pm 1.75$ & $3.20 \pm 0.69$ & $13.72 \pm 1.31$ & -0.97 & +0.34 & -0.44 & {$[129]$} \\
KLOE & $25.6 \pm 1.8$ & $1.5 \pm 0.8$ & $15.4 \pm 2.2$ & -0.95 & +0.29 & -0.38 & {$[132]$} \\
NA48 & $24.86 \pm 1.88$ & $1.11 \pm 0.74$ & $10.25 \pm 1.05$ & -0.93 & +0.38 & -0.51 & {$[130,133]$} \\
ISTRA+ & $24.80 \pm 1.56$ & $1.94 \pm 0.88$ & $16.71 \pm 1.20$ & -0.94 & +0.34 & -0.44 & {$[131,134,127]$} \\
\hline Our avg & $25.02 \pm 1.12$ & $1.57 \pm 0.48$ & $13.34 \pm 1.41$ & -0.950 & +0.376 & -0.573 & \\
Our avg, no NA48 $K_{\mu 3}$ & $25.04 \pm 0.82$ & $1.57 \pm 0.36$ & $15.90 \pm 0.79$ & -0.942 & +0.234 & -0.349 \\
\hline \hline
\end{tabular}

Table 10. Quadratic-linear form factor parameters for $K_{\ell 3}$ decays from KLOE, KTeV, NA48, and ISTRA+. For each experiment, the results for $K_{e 3}$ and $K_{\mu 3}$ decays are averaged. The input $K_{e 3}$ values from NA48 and all values from ISTRA+ have been converted for use with the notation of Eq. (28); correlation coefficients for these measurements were communicated privately. Our averages of the results from all four experiments, with and without the NA48 $K_{\mu 3}$ data included, are also listed.

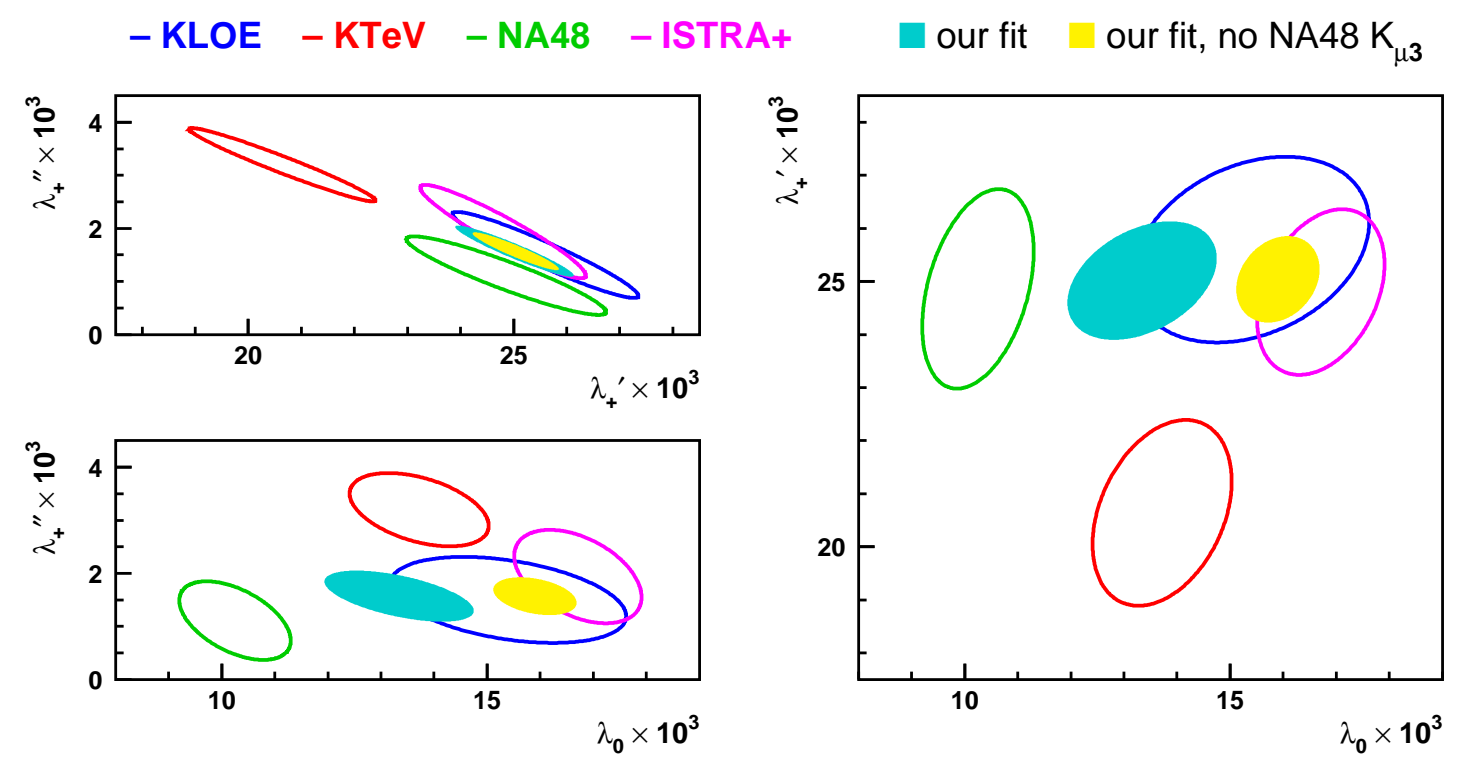

Fig. 6. $1 \sigma$ confidence contours for measurements of $\lambda_{+}^{\prime}, \lambda_{+}^{\prime \prime}$, and $\lambda_{0}$ from KLOE, KTeV, NA48, and ISTRA+. For each experiment, the results for $K_{e 3}$ and $K_{\mu 3}$ decays are averaged. Our averages of the results from all four experiments, with and without the NA48 $K_{\mu 3}$ data included, are also shown.

\begin{tabular}{lcccc}
\hline \hline Experiment & $\Lambda_{+} \times 10^{3}$ & $\ln C$ & $\rho$ & Ref. \\
\hline KLOE & $25.70 \pm 0.57$ & $0.2038 \pm 0.0241$ & -0.26 & {$[132$} \\
KTeV & $25.09 \pm 0.44$ & $0.1915 \pm 0.0116$ & -0.27 & {$[68$} \\
NA48 & $24.60 \pm 1.47$ & $0.1354 \pm 0.0133$ & -0.24 & {$[133$} \\
ISTRA+ & $26.13 \pm 0.52$ & $0.2084 \pm 0.0134$ & -0.46 & {$[127$} \\
\hline Average & $25.66 \pm 0.41$ & $0.2004 \pm 0.0091$ & -0.33 & \\
\hline \hline
\end{tabular}

Table 11. Dispersive form factor parameters for $K_{\ell 3}$ decays from KLOE, KTeV, NA48, and ISTRA+. For all experiments except NA48, the $K_{e 3}-K_{\mu 3}$ average is quoted by the experiment. NA48 does not quote an average; the table lists our average of their results, which has $S=2.0$ for $\Lambda_{+}$. The values from ISTRA+ have been converted for use with the notation of Eqs. (33) and (37); the systematic uncertainties on the ISTRA+ measurements are derived from systematic studies using the pole fits. Our average of the results from all four experiments, excluding the $K_{\mu 3}$ data from NA48, is also listed.

on the values from the individual experiments do not include the contributions arising from the representation of the form factor phase in the dispersive parameterization; these contributions are common to all experiments and are included as additional uncertainties of $0.30 \times 10^{-3}$ on our average value of $\Lambda_{+}$and 0.0040 on our average value of $\ln C$.

The use of the dispersive form factor parameterization clearly illustrates the contrast between the $K_{\mu 3}$ result from NA48 and those from the other experiments. The re- maining measurements are in agreement. Our average of the results from all four experiments, excluding only the $K_{\mu 3}$ data from NA48, is plotted as the yellow ellipse. This average gives $\chi^{2} / \mathrm{ndf}=5.6 / 5(P=34.4 \%)$. By contrast, if the $K_{\mu 3}$ data from NA48 are included, the average gives $\chi^{2} /$ ndf $=25.7 / 6(P=0.026 \%)$. On this basis, we exclude the NA48 $K_{\mu 3}$ form factor results from the averages used to calculate the phase space integrals for the evaluation of $\left|V_{u s}\right|$ and related tests. 


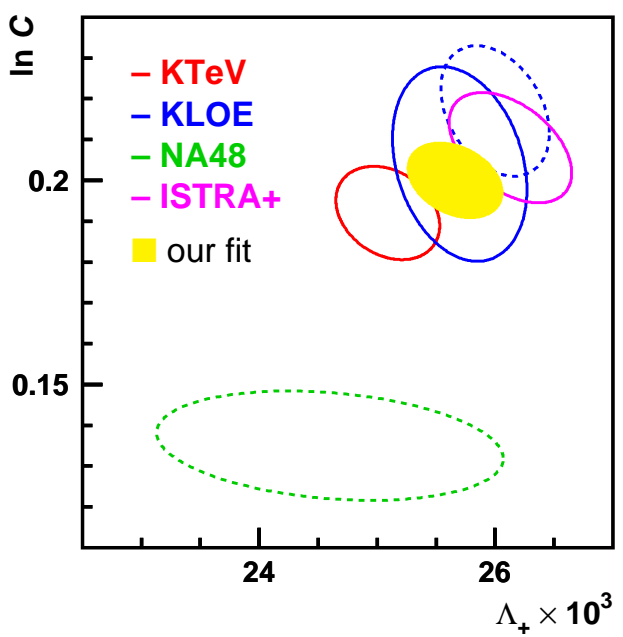

Fig. 7. $1 \sigma$ confidence contours for measurements of $\Lambda_{+}$and $\ln C$ from KLOE, KTeV, NA48, and ISTRA+. For each experiment, the results for $K_{e 3}$ and $K_{\mu 3}$ decays are averaged. Our average of the results from all four experiments, excluding the $K_{\mu 3}$ data from NA48, is also shown. A new, preliminary KLOE result 136 is shown as the dashed blue ellipse. This is not included in the fit.

\begin{tabular}{lccc}
\hline \hline Integral & $\lambda_{+}^{\prime}, \lambda_{+}^{\prime \prime}, \lambda_{0}$ & $\Lambda_{+}, \ln C$ & Rel. diff. \\
\hline$I\left(K_{e 3}^{0}\right)$ & $0.15457(20)$ & $0.15476(18)$ & $+0.12 \%$ \\
$I\left(K_{e 3}^{ \pm}\right)$ & $0.15894(21)$ & $0.15922(18)$ & $+0.18 \%$ \\
$I\left(K_{\mu 3}^{0}\right)$ & $0.10266(20)$ & $0.10253(16)$ & $-0.13 \%$ \\
$I\left(K_{\mu 3}^{ \pm}\right)$ & $0.10564(20)$ & $0.10559(17)$ & $-0.05 \%$ \\
$\rho\left(K_{e 3}, K_{\mu 3}\right)$ & +0.56 & +0.38 & \\
\hline \hline
\end{tabular}

Table 12. Comparison of phase space integrals evaluated from our averages of the results of quadratic-linear $\left(\lambda_{+}^{\prime}, \lambda_{+}^{\prime \prime}, \lambda_{0}\right)$ and dispersive $\left(\Lambda_{+}, \ln C\right)$ fits.

Table 12 lists the values of the phase space integrals as computed from the results of our averages of the experimental form factor data using the quadratic-linear and dispersive parameterizations. (In the case of the dispersive parameterization, the uncertainties arising from the representation of the form factor phase are included in the overall uncertainty for each integral.) For both parameterizations, the correlations between the uncertainties on the integrals are described by a matrix of the form

$$
\left(\begin{array}{llll}
1 & 1 & \rho & \rho \\
1 & 1 & \rho & \rho \\
\rho & \rho & 1 & 1 \\
\rho & \rho & 1 & 1
\end{array}\right) .
$$

The table lists the values of the correlation coefficient $\rho$ for each parameterization.

As seen in the table, when evaluated from the dispersive fit results, the integrals tend to be very slightly different than they are when evaluated from the quadraticlinear fit results. Nowhere is this difference greater than $0.2 \%$. As expected, the $K_{\mu 3}$ integrals are slightly smaller when obtained from the dispersive fit results (Sect. 2.2.4). Given the advantages of the dispersive parameterization,

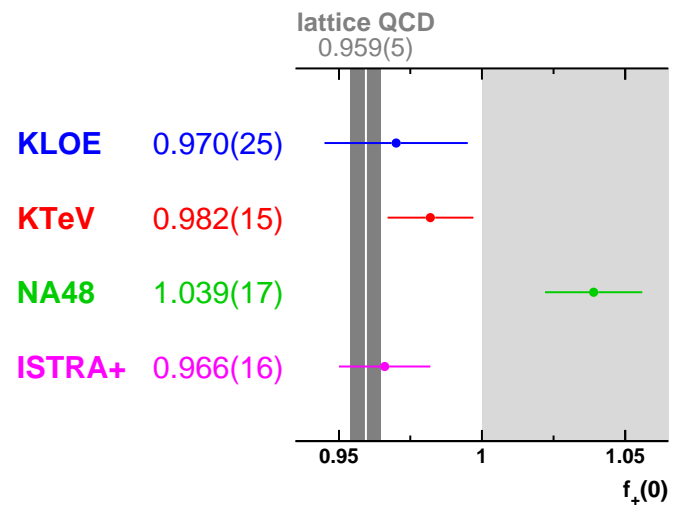

Fig. 8. Values for $f_{+}(0)$ from measurements of $\ln C$ from different experiments, using the Callan-Treiman relation and $f_{K} / f_{\pi}=1.193(6)$. The lattice QCD result $f_{+}(0)=0.959(5)$ is also shown.

we use the integrals calculated from the dispersive fit results for the evaluation of $\left|V_{u s}\right|$ and related tests.

\subsubsection{The $K_{\mu 3}$ scalar form factor and tests of chiral perturbation theory}

Given a value for $f_{K} / f_{\pi}$, the Callan-Treiman relation (Eq. (31) ) can be used to obtain a value for $f_{+}(0)$ from a measurement of $\ln C$, providing a test of consistency between scalar form factor measurements and lattice calculations. Figure 8 shows the values for $f_{+}(0)$ corresponding to the measurements of $\ln C$ from different experiments, using the CT relation and $f_{K} / f_{\pi}=1.193(6)$ (Sect. 2.1.1). For this exercise, the uncertainties on each value of $\ln C$ include the common contribution from the parameterization of the form factor phase. The lattice QCD value, $f_{+}(0)=0.959(5)$ (Eq. (17)), is also shown. The measurements of $\ln C$ from KLOE, $\mathrm{KTeV}$, and ISTRA+ give values for $f_{+}(0)$ that are essentially consistent with the lattice estimate, although they tend to be a little larger. The NA48 result, on the other hand, gives $f_{+}(0)=1.039(17)$, which is in contrast with the theoretical expectation of Fubini and Furlan that $f_{+}(0)<1$ [137]. As noted in Sect. 3.5.2 we exclude the NA48 $K_{\mu 3}$ form factor measurements from the averages used to calculate the phase space integrals for the evaluation of $\left|V_{u s}\right|$ and related tests. Our resulting world-average values for $\Lambda_{+}$and $\ln C$ are listed in Table 11. The value $\ln C=0.2004(91)$, when used with $f_{K} / f_{\pi}=1.193(6)$, gives $f_{+}(0)=0.974(12)$. More generally, the experimental data on $\ln C$ alone give $\left(f_{K} / f_{\pi}\right) / f_{+}(0)=1.225(14)$. This result is completely independent of any information from lattice estimates.

Alternatively, one can perform a fit to the world-average value of $\ln C$, together with the lattice determinations $f_{K} / f_{\pi}=1.193(6)$ and $f_{+}(0)=0.959(5)$, using the constraint given by the CT relation. When performing such a fit, we make use of a recent preliminary measurement from KLOE [136] of the dispersive $K_{\mu 3}$ form factor parameters. This measurement, which is illustrated as the dashed ellipse in Fig. 7, is used here in place of the pub- 


\begin{tabular}{lc|ccc}
\hline \hline Parameter & Value & \multicolumn{2}{|c}{ Corr. matrix (\%) } \\
\hline$\Lambda_{+} \times 10^{3}$ & $25.7 \pm 0.5$ & -27 & +10 & -10 \\
$\ln C$ & $0.208(8)$ & & -38 & +38 \\
$f_{+}(0)$ & $0.961(5)$ & & & +19 \\
$f_{K} / f_{\pi}$ & $1.191(6)$ & & & \\
\hline \hline
\end{tabular}

Table 13. Results of a fit to the experimental average value for $\ln C$ (including a preliminary KLOE measurement) and lattice results for $f_{+}(0)$ and $f_{K} / f_{\pi}$, using the constraint provided by the Callan-Treiman theorem.

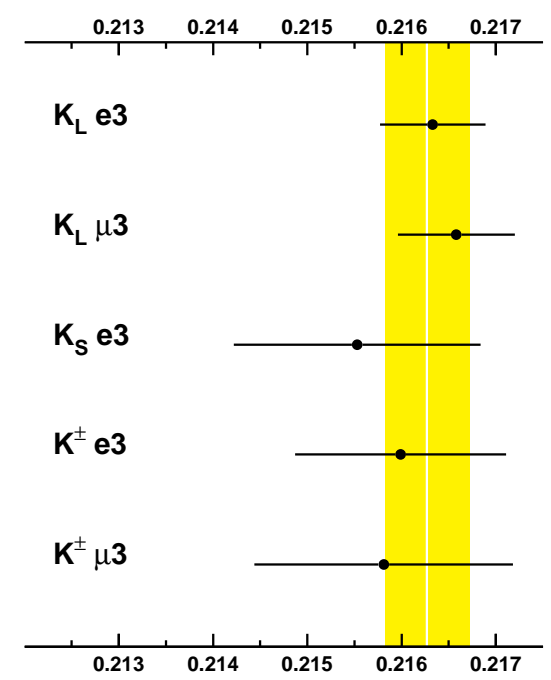

Fig. 9. Comparison of values for $\left|V_{u s}\right| f_{+}(0)$ for all channels. Our average is indicated by the yellow band.

lished KLOE measurement in Table11, Although preliminary, we make use of this measurement here as we are only interested in demonstrating the power of the consistency test offered by the CT relation. The average of the dispersive $K_{\mu 3}$ form factor parameters using the new KLOE measurement gives $\Lambda_{+}=(25.78 \pm 0.40) \times 10^{-3}$ and $\ln C=$ $0.2034(86)$, with $\rho\left(\Lambda_{+}, \ln C\right)=-0.34$ and $\chi^{2} / \mathrm{ndf}=7.4 / 5$ $(P=19.2 \%)$. The value of $\ln C$ is then fitted together with the lattice inputs as described above to obtain the results in Table 13] The fit gives $\chi^{2} / \mathrm{ndf}=0.78 / 1(P=38 \%)$, confirming the agreement between the experimental measurements of $\ln C$ and the lattice determinations of $f_{+}(0)$ and $f_{K} / f_{\pi}$. The values of $f_{+}(0)$ and $f_{K} / f_{\pi}$ move only slightly, and the uncertainties on these values are slightly decreased.

\section{Physics results}

\subsection{Determination of $\left|V_{u s}\right| f_{+}(0)$}

For each of the five decay modes for which rate measurements exist, we use Eq. (9) to evaluate $\left|V_{u s}\right| f_{+}(0)$ from the decay rate data in Tables 3 , 4, and 6, the phase space integrals from dispersive fits in Table 12, the long-distance radiative corrections in Table 1, and the $\mathrm{SU}(2)$-breaking corrections of [49] discussed in Sect. 2.2.3. We keep track of the correlations between the uncertainties on the values of $\left|V_{u s}\right| f_{+}(0)$ from different modes arising from the use of common corrections and from correlations in the input data set (e.g., from the outputs of the fits to BR and lifetime measurements). The resulting values of $\left|V_{u s}\right| f_{+}(0)$ are listed in Table 14 and illustrated in Fig. 9, The principal experimental result of this review is the average value

$$
\left|V_{u s}\right| f_{+}(0)=0.2163(5),
$$

which has an uncertainty of about of $0.2 \%$. The results from the five modes are in good agreement; the fit gives $\chi^{2} /$ ndf $=0.77 / 4(P=94 \%)$. Table 14 gives an approximate breakdown of the sources contributing to the total uncertainty on the determination of $\left|V_{u s}\right| f_{+}(0)$ from each mode. The best single determinations of $\left|V_{u s}\right| f_{+}(0)$ are from the $K_{e 3}$ and $K_{\mu 3}$ modes, with $K_{e 3}$ giving the slightly better determination since there is no contribution from uncertainties on the parameters of the scalar form factor. The limited precision of the value for $\left|V_{u s}\right| f_{+}(0)$ from $K_{e 3}$ decays of the $K_{S}$ decays is entirely determined by the experimental uncertainty on the corresponding $\mathrm{BR}$, which is dominantly statistical. A better measurement of the BR for this decay would allow knowledge of $\left|V_{u s}\right| f_{+}(0)$ to be significantly improved, since $\tau_{K_{S}}$ is known very precisely. The values of $\left|V_{u s}\right| f_{+}(0)$ from charged kaon decays are currently limited in precision both by experimental uncertainties in the corresponding BRs and by the uncertainty in the theoretical estimate of $\delta_{\mathrm{SU}(2)}^{K^{+} \pi^{0}}$.

\subsection{Accuracy of isospin-breaking corrections}

The average values for $\left|V_{u s}\right| f_{+}(0)$ can be computed separately for charged and neutral kaon decays. The ratio of these values (calculated without applying $\mathrm{SU}(2)$-breaking corrections) then gives an experimental estimate of $\delta_{\mathrm{SU}(2)}^{K^{+} \pi^{0}}$ for comparison with the estimate from chiral perturbation theory. We obtain

$$
\delta_{\mathrm{SU}(2), \exp }^{K^{+}}=0.027 \pm 0.004,
$$

in good agreement with the theoretical estimate of Ref.49, $\delta_{\mathrm{SU}(2)}^{K^{+} \pi^{0}}=0.029 \pm 0.004$ (Eq. (26) $)$. We observe that the uncertainty on the theoretical value of $\delta_{\mathrm{SU}(2)}^{K^{+} \pi^{0}}$ contributes significantly to the overall uncertainties on the values of $\left|V_{u s}\right| f_{+}(0)$ for charged kaon decay modes (see Table 14).

\subsection{Lepton universality}

Comparison of the values of $\left|V_{u s}\right| f_{+}(0)$ computed separately for $K_{e 3}$ and $K_{\mu 3}$ modes provides a test of lepton universality. Specifically,

$$
r_{\mu e}=\frac{\left[\left|V_{u s}\right| f_{+}(0)\right]_{\mu 3, \exp }^{2}}{\left[\left|V_{u s}\right| f_{+}(0)\right]_{e 3, \exp }^{2}}=\frac{\Gamma_{K \mu 3}}{\Gamma_{K e 3}} \frac{I_{e 3}\left(1+2 \delta_{\mathrm{EM}}^{K e}\right)}{I_{\mu 3}\left(1+2 \delta_{\mathrm{EM}}^{K \mu}\right)} .
$$

$\left(\right.$ Here, $\delta_{\mathrm{EM}}^{K e}$ and $\delta_{\mathrm{EM}}^{K \mu}$ are the electromagnetic corrections for kaons of the charge state under consideration.) By 


\begin{tabular}{|c|c|c|c|c|c|c|c|c|c|c|}
\hline Mode & $\left|V_{u s}\right| f_{+}(0)$ & $\%$ err & $\overline{\mathrm{BR}}$ & $\bar{\tau}$ & $\Delta$ & Int & \multicolumn{4}{|c|}{ Correlation matrix (\%) } \\
\hline$K_{L} \rightarrow \pi e \nu$ & $0.2163(6)$ & 0.26 & 0.09 & 0.20 & 0.11 & 0.06 & +55 & +10 & +3 & 0 \\
\hline$K_{L} \rightarrow \pi \mu \nu$ & $0.2166(6)$ & 0.29 & 0.15 & 0.18 & 0.11 & 0.08 & & +6 & 0 & +4 \\
\hline$K_{S} \rightarrow \pi e \nu$ & $0.2155(13)$ & 0.61 & 0.60 & 0.03 & 0.11 & 0.06 & & & +1 & 0 \\
\hline$K^{ \pm} \rightarrow \pi e \nu$ & $0.2160(11)$ & 0.52 & 0.31 & 0.09 & 0.40 & 0.06 & & & & +73 \\
\hline$K^{ \pm} \rightarrow \pi \mu \nu$ & $0.2158(14)$ & 0.63 & 0.47 & 0.08 & 0.39 & 0.08 & & & & \\
\hline Average & $0.2163(5)$ & & & & & & & & & \\
\hline
\end{tabular}

Table 14. Values of $\left|V_{u s}\right| f_{+}(0)$ as determined from each kaon decay mode, with approximate contributions to relative uncertainty (\% err) from branching ratios (BR), lifetimes $(\tau)$, combined effect of $\delta_{\mathrm{EM}}^{K \ell}$ and $\delta_{\mathrm{SU}(2)}^{K \ell}(\Delta)$, and phase space integrals (Int).

comparison with Eq. (9), $r_{\mu e}$ is equal to the ratio $g_{\mu}^{2} / g_{e}^{2}$, with $g_{\ell}$ the coupling strength at the $W \rightarrow \ell \nu$ vertex. In the SM, $r_{\mu e}=1$.

Before the advent of the new BR measurements described in Sects. 3.2 and 3.4 the values of $\left|V_{u s}\right| f_{+}(0)$ from $K_{e 3}$ and $K_{\mu 3}$ rates were in substantial disagreement. Using the $K_{L}$ and $K^{ \pm}$BRs from the 2004 edition of the PDG compilation [100] (and assuming current values for the $I_{\ell 3}$ and $\left.\delta_{\mathrm{EM}}^{K \ell}\right)$, we obtain $r_{\mu e}=1.013(12)$ for $K^{ \pm}$decays and 1.040 (13) for $K_{L}$ decays.

As noted in Sect. 3.2 the new BR measurements procure much better agreement. From the entries in Table 14 we calculate $r_{\mu e}$ separately for charged and neutral modes (including the value of $\left|V_{u s}\right| f_{+}(0)$ from $K_{S} \rightarrow \pi e \nu$ decays, though this has little impact) and obtain $0.998(9)$ and 1.003(5), respectively. The results are compatible; the average value is $r_{\mu e}=1.002(5)$. As a statement on the lepton-flavor universality hypothesis, we note that the sensitivity of this test approaches that obtained with $\pi \rightarrow$ $\ell \nu$ decays $\left(\left(r_{\mu e}\right)=1.0042(33)\right.$ [138] $)$ and $\tau \rightarrow \ell \nu \bar{\nu}$ decays $\left(\left(r_{\mu e}\right)=1.000(4)\right.$ [139]). Alternatively, if the leptonuniversality hypothesis is assumed to be true, the equivalence of the values of $\left|V_{u s}\right| f_{+}(0)$ from $K_{e 3}$ and $K_{\mu 3}$ demonstrates that the calculation of the long-distance corrections $\delta_{\mathrm{EM}}^{K \ell}$ is accurate to the per-mil level.

\subsection{Determination of $\left|V_{u s} / V_{u d}\right| \times f_{K} / f_{\pi}$}

As noted in Sect. 2.1, Eq. (2) allows the ratio $\left|V_{u s} / V_{u d}\right| \times$ $f_{K} / f_{\pi}$ to be determined from experimental information on the radiation-inclusive $K_{\ell 2}$ and $\pi_{\ell 2}$ decay rates. The limiting uncertainty is that from $\operatorname{BR}\left(K_{\mu 2(\gamma)}\right)$, which is $0.28 \%$ as per Table 6. Using this, together with the value of $\tau_{K^{ \pm}}$ from the same fit and $\Gamma\left(\pi^{ \pm} \rightarrow \mu^{ \pm} \nu\right)=38.408(7) \mu \mathrm{s}^{-1}$ [87] we obtain

$$
\left|V_{u s} / V_{u d}\right| \times f_{K} / f_{\pi}=0.2758(5) .
$$

\subsection{Test of CKM unitarity}

We determine $\left|V_{u s}\right|$ and $\left|V_{u d}\right|$ from a fit to the results obtained above. As starting points, we use the value $\left|V_{u s}\right| f_{+}(0)=0.2163(5)$ given in Table 14, together with the lattice QCD estimate $f_{+}(0)=0.959(5)$ (Eq. (17)). We also use the result $\left|V_{u s} / V_{u d}\right| \times f_{K} / f_{\pi}=0.2758(5)$

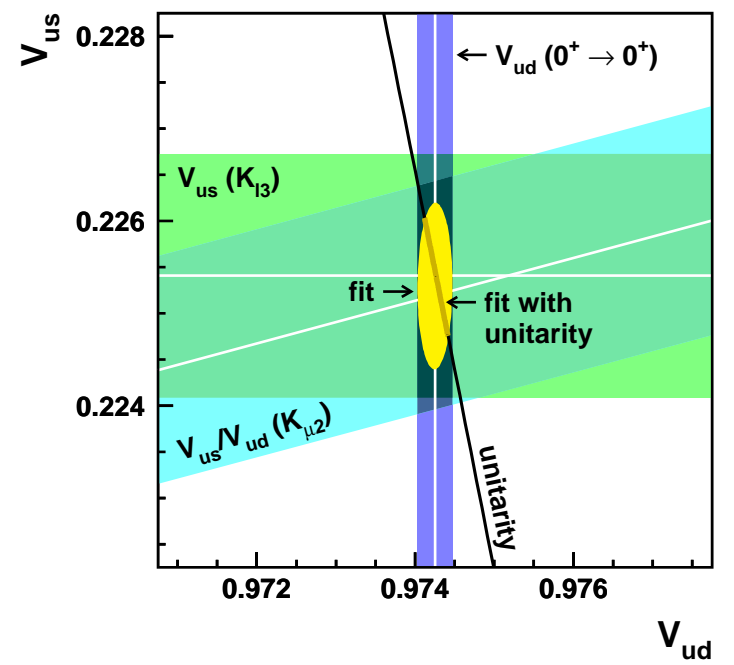

Fig. 10. Results of fits to $\left|V_{u d}\right|,\left|V_{u s}\right|$, and $\left|V_{u s} / V_{u d}\right|$.

discussed in Sect. 4.4 together with the lattice estimate $f_{K} / f_{\pi}=1.193(6)$ (Sect. 2.1.1). Thus we have

$$
\begin{aligned}
\left|V_{u s}\right| & =0.2254(13) & & {\left[K_{\ell 3} \text { only }\right] } \\
\left|V_{u s} / V_{u d}\right| & =0.2312(13) & & {\left[K_{\ell 2} \text { only }\right] . }
\end{aligned}
$$

Finally, we use the evaluation $\left|V_{u d}\right|=0.97425(22)$ from a recent survey 140, of half-life, decay-energy, and BR measurements related to 20 superallowed $0^{+} \rightarrow 0^{+}$nuclear beta decays, which includes a number of new, highprecision Penning-trap measurements of decay energies, as well as the use of recently improved electroweak radiative corrections [141] and new isospin-breaking corrections [142, in addition to other improvements over past surveys by the same authors. Our fit to these inputs gives

$$
\begin{aligned}
& \left|V_{u d}\right|=0.97425(22), \\
& \left|V_{u s}\right|=0.2253(9) \quad\left[K_{\ell 3}, K_{\ell 2}, 0^{+} \rightarrow 0^{+}\right],
\end{aligned}
$$

with $\chi^{2} /$ ndf $=0.014 / 1(P=91 \%)$ and negligible correlation between $\left|V_{u d}\right|$ and $\left|V_{u s}\right|$. With the current worldaverage value, $\left|V_{u b}\right|=0.00393(36)$ [87, the first-row unitarity sum is then $\Delta_{\mathrm{CKM}}=\left|V_{u d}\right|^{2}+\left|V_{u s}\right|^{2}+\left|V_{u b}\right|^{2}-1=$ $-0.0001(6)$; the result is in striking agreement with the unitarity hypothesis. (Note that the contribution to the sum from $\left|V_{u b}\right|$ is essentially negligible.) As an alternate expression of this agreement, we may state a value for 


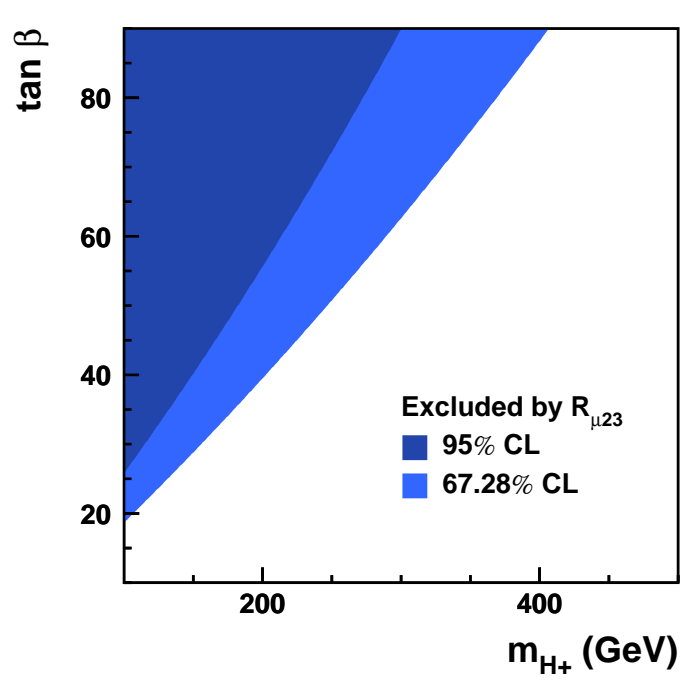

Fig. 11. Regions in the $\left(m_{H^{ \pm}}, \tan \beta\right)$ plane in two-Higgsdoublet models excluded by the present result for $R_{\mu 23}$.

$$
\begin{array}{r}
G_{\mathrm{CKM}}=G_{\mu} \sqrt{\left|V_{u d}\right|^{2}+\left|V_{u s}\right|^{2}+\left|V_{u b}\right|^{2}} \text {. We obtain } \\
G_{\mathrm{CKM}}=1.16633(35) \times 10^{-5} \mathrm{GeV}^{-2}
\end{array}
$$

with $G_{\mu}=1.166371(6) \times 10^{-5} \mathrm{GeV}^{-2} 3$.

It is also possible to perform the fit with the unitarity constraint included, increasing by one the number of degrees of freedom. The constrained fit gives

$$
\left|V_{u s}\right|=\sin \theta_{C}=\lambda=0.2254(6) \quad \text { [with unitarity] }
$$

and $\chi^{2} /$ ndf $=0.024 / 2(P=99 \%)$. This result and that obtained above without assuming unitarity are both illustrated in Fig. 10.

At this point, using Eq. (47) and the phenomenological value $\Delta_{\mathrm{CKM}}=-0.0001(6)$, it is possible to set bounds on the effective scale of the four operators that parameterize new physics contributions to $\Delta_{\mathrm{CKM}}$. We obtain

$$
\Lambda>11 \mathrm{TeV} \quad \text { (90\% C.L.). }
$$

As noted in Ref. 76, for the operators $O_{l l}^{(3)}, O_{\varphi l}^{(3)}$, and $O_{\varphi q}^{(3)}$ (see Eqs. (46)), this constraint is at the same level as the constraints from $Z$-pole measurements. For the four-fermion operator $O_{l q}^{(3)}, \Delta_{\mathrm{CKM}}$ improves upon existing bounds from LEP2 by an order of magnitude.

\subsection{Bounds on non-helicity-suppressed amplitudes}

As noted in Sect. 2.3.3, an empirical value for the ratio $R_{\mu 23}$ (Eq. (50)) can be used to exclude regions of the $\left(m_{H^{ \pm}}, \tan \beta\right)$ parameter space in models with two Higgs doublets, such as the minimal supersymmetry extension of the SM (Eq. (51)). Operatively, we evaluate $R_{\mu 23}$ via a fit akin to that described in Sect. 4.5, but with separate parameters accounting for the values of $\left|V_{u s}\right|$ from $K_{\ell 3}$ and $K_{\mu 2}$ decays. The fit then has three free parameters: the value of $\left|V_{u s}\right|$ from $K_{\ell 3}$ decays, the value of

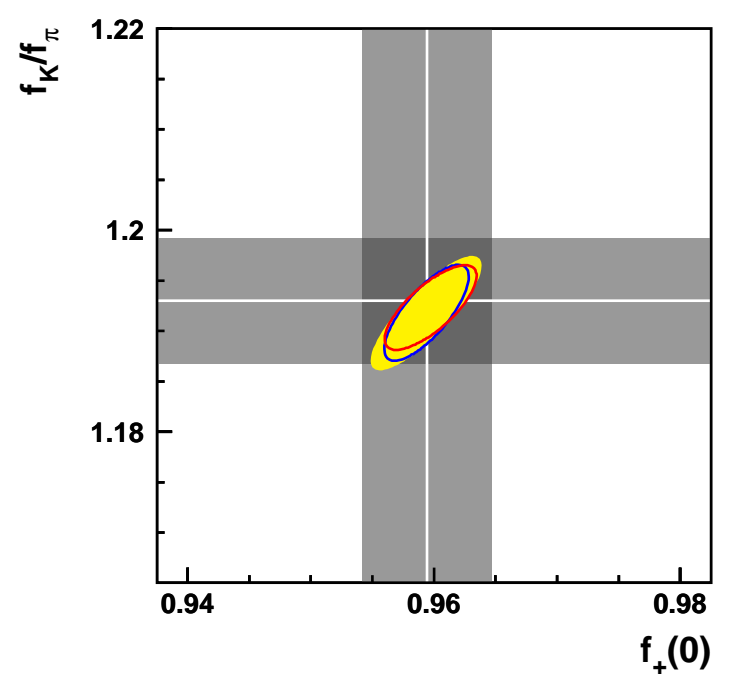

Fig. 12. Results of fit for $f_{+}(0)$ and $f_{K} / f_{\pi}$, given experimental data and first-row CKM unitarity: (yellow) no input from lattice; (blue) $f_{+}(0)=0.959(5)$ as input to the fit and no input for $f_{K} / f_{\pi}$; (red) $f_{K} / f_{\pi}=1.193(6)$ as input and no input for $f_{+}(0)$. The grey bands illustrate the reference values for $f_{+}(0)$ and $f_{K} / f_{\pi}$.

$\left|V_{u s} / V_{u d}\right|$ from $K_{\mu 2}$ decays, and the value of $\left|V_{u d}\right|$ from $0^{+} \rightarrow 0^{+}$nuclear beta decays. The input values used for $\left|V_{u s}\right|$ and $\left|V_{u s} / V_{u d}\right|$ are from Eq. (56) and include the relevant lattice constants. The contribution to non-helicitysuppressed $K_{\ell 3}$ decays from charged Higgs exchange is negligible, so we include as a constraint in the fit the firstrow unitarity condition on the value of $\left|V_{u s}\right|$ from $K_{\ell 3}$ decays: $\left|V_{u d}\right|^{2}+\left|V_{u s}\right|_{K_{\ell 3}}^{2}+\left|V_{u b}\right|^{2}=1$. Expressing the results of the fit in terms of $\left|V_{u s}\right|$ from $K_{\ell 3}$ decays and the ratio $R_{\mu 23}$, we obtain

$$
\begin{aligned}
& \left|V_{u s}\right|=0.2254(8) \quad\left[K_{\ell 3}, 0^{+} \rightarrow 0^{+}, \text {unitarity }\right] \\
& R_{\mu 23}=0.999(7) \quad\left[K_{\mu 2}\right] .
\end{aligned}
$$

The fit gives $\chi^{2} / \mathrm{ndf}=0.0003 / 1(P=99 \%)$, with $\rho=$ -0.55 between the parameter uncertainties in the stated basis. The regions of the $\left(m_{H^{ \pm}}, \tan \beta\right)$ parameter space in models with two Higgs doublets excluded at the $1 \sigma$ and 95\% CLs by this result for $R_{\mu 23}$ are shown as the shaded area in Fig. 11. The bound is obtained setting $\epsilon_{0}=1 / 16 \pi^{2}$ in Eq. (51), as expected in the MSSM. Note that this result excludes the region at low $m_{H^{ \pm}}$and large $\tan \beta$ favoured by $B \rightarrow \tau \nu[143$.

\subsection{Determination of Standard Model values for $f_{+}(0)$ and $f_{K} / f_{\pi}$ from experimental data}

Equation (2), which in the SM relates the ratio of $K_{\ell 2}$ and $\pi_{\ell 2}$ decay rates to the ratio $\left|V_{u s} / V_{u d}\right| \times f_{K} / f_{\pi}$, can be rewritten

$$
Q_{\ell 2}=\frac{\left(\left|V_{u s}\right| f_{+}(0)\right)^{2}}{\left|V_{u d}\right|^{2}} \times \frac{1}{f_{+}(0)^{2}} \times \frac{f_{K}^{2}}{f_{\pi}^{2}},
$$


where $Q_{\ell 2}$ is the ratio of $\Gamma\left(K_{\ell 2(\gamma)}\right)$ to $\Gamma\left(\pi_{\ell 2(\gamma)}\right)$ with phase space differences factored out and radiative corrections applied. As per the discussion in Sects. 2.1 and 4.4, for $K_{\mu 2(\gamma)}$ and $\pi_{\mu 2(\gamma)}$ decays, $Q_{\mu 2}=0.07604(26)$.

Equation (62) can be used together with the experimentally derived values for $Q_{\mu 2}$ from $K_{\mu 2}$ and $\pi_{\mu 2}$ rates, $\left|V_{u s}\right| f_{+}(0)$ from $K_{\ell 3}$ decays, and $\left|V_{u d}\right|$ from $0^{+} \rightarrow 0^{+}$nuclear beta decays to determine the values of the hadronic constants $f_{+}(0)$ and $f_{K} / f_{\pi}$. A straightforward calculation gives

$$
\left(f_{K} / f_{\pi}\right) / f_{+}(0)=1.242(4) .
$$

This value essentially depends only on experimental $K_{\mu 2}$ and $\pi_{\mu 2}$ rate data and radiative corrections. CKM unitarity is not assumed, although the equality of $\left|V_{u s}\right|$ in $K_{\mu 2}$ and $K_{\ell 3}$ decays is. If we further assume the validity of our reference lattice value $f_{+}(0)=0.959(5)$, we obtain $f_{K} / f_{\pi}=1.192(8)$, while if we assume the validity of our reference value $f_{K} / f_{\pi}=1.193(6)$, we obtain $f_{+}(0)=0.960(6)$.

To go further, we impose first-row CKM unitarity as an additional constraint. We can then perform a fit with $Q_{\mu 2}$, $\left|V_{u s}\right| f_{+}(0),\left|V_{u d}\right|, f_{+}(0)$, and $f_{K} / f_{\pi}$ as parameters, and two constraints: $\left|V_{u d}\right|^{2}+\left|V_{u s}\right|^{2}+\left|V_{u b}\right|^{2}=1$ and Eq. (62). Up to two of the parameters can be left free for a solution with no degrees of freedom. Taking these to be $f_{+}(0)$ and $f_{K} / f_{\pi}$, we obtain

$$
\begin{aligned}
f_{+}(0) & =0.959(5), \\
f_{K} / f_{\pi} & =1.192(6) \quad \text { [with unitarity], }
\end{aligned}
$$

with a correlation coefficient of $\rho=+0.84$. This solution is illustrated as the yellow ellipse in Fig. 12. Once again, we note that this result is obtained without any recourse to prior estimates of $f_{+}(0)$ or $f_{K} / f_{\pi}$. The lattice values of these constants are illustrated as the grey bands in Fig. 12 for the purposes of comparison only.

With either of the reference values of $f_{+}(0)$ or $f_{K} / f_{\pi}$ as an additional input, the fit has one degree of freedom. With $f_{+}(0)=0.959(5)$ as input, the fit gives $\left|V_{u d}\right|=$ $0.97425(18),\left|V_{u s}\right| f_{+}(0)=0.2163(4), f_{+}(0)=0.9594(34)$, and $f_{K} / f_{\pi}=1.192(5)$, with $\rho=+0.76$ the correlation coefficient between $f_{+}(0)$ and $f_{K} / f_{\pi}$, and $\chi^{2}=3 \times 10^{-4} / 1$ $(P=99 \%)$. This is illustrated as the blue ellipse in Fig.12. With $f_{K} / f_{\pi}=1.193(6)$ as input instead, the fit gives $\left|V_{u d}\right|=0.97427(17),\left|V_{u s}\right| f_{+}(0)=0.2163(5), f_{+}(0)=$ $0.960(4)$, and $f_{K} / f_{\pi}=1.192(4)$, with $\rho=+0.75$ and $\chi^{2}=0.023 / 1(P=88 \%)$. This is illustrated as the red ellipse in Fig. 12, In summary, our chosen reference values for $f_{+}(0)$ and $f_{K} / f_{\pi}$ are a near-perfect match with experimental data and SM assumptions.

\section{Conclusions}

At present, the experimental precision of data on leptonic and semileptonic kaon decays is nicely matched below the percent level by the theoretical precision, allowing us to perform very precise measurements of SM parameters and to set stringent bounds on beyond-SM physics. In this work, we have presented an updated analysis of the following three main subjects: (i) the overall determination of $\left|V_{u s}\right|$, with and without imposing CKM unitarity; (ii) the comparison between the values of $\left|V_{u s}\right|$ as determined from $K_{\ell 3}$ and $K_{\mu 2}$ decay data, and the corresponding constraints on deviations from the $V-A$ structure; (iii) tests of lepton universality in $K_{\ell 3}$ decays. The final results of our analysis are presented in detail in Sect. 4 and will not be repeated here. As a general conclusion, we simply emphasize that the $\mathcal{O}(10 \mathrm{TeV})$ bound on the scale of new physics (Eq. (60) ), which follows from the verification of the first-row CKM unitarity condition, represents one of the most stringent constraints on physics beyond the Standard Model.

In addition to the three main topics mentioned above, our analysis has allowed some interesting cross-checks of both analytic and lattice-based theoretical results relevant to kaon physics, most notably the determination of the mass-induced isospin-breaking effect between charged and neutral semileptonic kaon decays (Eq. (53)) and the indirect determination of the kaon form factors shown in Fig. 12, We emphasize that both of these results, which agree well with the current theoretical estimates, are strictly valid only under a pure SM hypothesis.

\section{Acknowledgments}

The authors thank P. Franzini and all other members of the FlaviaNet Kaon Working Group, as well as V. Lubicz and A. Jüttner of the FlaviaNet Lattice Averaging Group (FLAG), for useful discussion, comments, and suggestions. This work is supported by the European Union Sixth Framework Programme under contract MTRN-CT-2006035482, FlaviaNet. E.P. acknowledges financial support from MEC (Spain) under grant FPA2007-60323. F.M. acknowledges financial support from projects FPA200766665, 2009SGR502. E.P. and F.M. acknowledge support from the Spanish Consolider-Ingenio 2010 Programme CPAN (CSD2007-00042). G.I. acknowledges support from the Technische Universität München - Institute for Advanced Study, funded by the German Excellence Initiative.

\section{A Polynomial approximations for the phase space integrals $I_{K \ell}$}

In this appendix, we give the explicit forms used to evaluate the phase space integrals $I_{K \ell}$ using the form factor parameters obtained from fits with different parameterizations. In all cases, we insert the expressions for $\bar{f}_{+, 0}(t)$ into the integrand of Eq. (27) and obtain a polynomial expression in the form factor parameters for the integral $I_{K \ell}$, with coefficients depending on the mass values $m_{K}$ and $m_{\ell}$. 
The form of the phase space integral used with the polynomial parameterization up to cubic terms in $t$ is

$$
\begin{aligned}
I_{K \ell} & =z_{0}+z_{1} \lambda_{+}^{\prime}+z_{2}\left(\lambda_{+}^{\prime 2}+\lambda_{+}^{\prime \prime}\right)+z_{3} \lambda_{+}^{\prime} \lambda_{+}^{\prime \prime}+z_{4} \lambda_{+}^{\prime \prime 2} \\
& +z_{5} \lambda_{+}^{\prime \prime \prime}+z_{6} \lambda_{+}^{\prime} \lambda_{+}^{\prime \prime \prime}+z_{7} \lambda_{+}^{\prime \prime} \lambda_{+}^{\prime \prime \prime}+z_{8} \lambda_{+}^{\prime \prime \prime 2} \\
& +z_{9} \lambda_{0}^{\prime}+z_{10}\left(\lambda_{0}^{\prime 2}+\lambda_{0}^{\prime \prime}\right)+z_{11} \lambda_{0}^{\prime} \lambda_{0}^{\prime \prime}+z_{12} \lambda_{0}^{\prime \prime 2} \\
& +z_{13} \lambda_{0}^{\prime \prime \prime}+z_{14} \lambda_{0}^{\prime} \lambda_{0}^{\prime \prime \prime}+z_{15} \lambda_{0}^{\prime \prime} \lambda_{0}^{\prime \prime \prime}+z_{16} \lambda_{0}^{\prime \prime \prime 2} .
\end{aligned}
$$

The numerical values of the coefficients $z$ are listed in Table 15. For $K_{e 3}$ decays, we take $z_{9} \ldots z_{17}$ to be zero. We also use the above form with to evaluate phase space integrals from the results of fits using the pole parameterization. In this case, we first perform a Taylor expansion of Eq. 29) up to cubic terms.

The form of the phase space integral used with the dispersive parameterizations of Eqs. (33) and (37) is

$$
\begin{aligned}
I_{K \ell} & =c_{0}+c_{1} \Lambda_{+}+c_{2} \Lambda_{+}^{2}+c_{3} \Lambda_{+}^{3}+c_{4} \Lambda_{+}^{4} \\
& +c_{5} \ln C+c_{6}(\ln C)^{2}+c_{7}(\ln C)^{3}+c_{8}(\ln C)^{4} .
\end{aligned}
$$

The numerical values of the coefficients $c$ are listed in Table 16. Again, for $K_{e 3}$ decays, we take $c_{5} \ldots c_{8}$ to be zero.

\section{References}

1. N. Cabibbo, Phys. Rev. Lett. 10 (1963) 531.

2. M. Kobayashi, T. Maskawa, Prog. Theor. Phys. 49 (1973) 652 .

3. MuLan Collaboration, D. Chitwood, et al., Phys. Rev. Lett. 99 (2007) 032001.

4. FlaviaNet Working Group on Kaon Decays, M. Antonelli, et al., arXiv:0801.1817 (2008).

5. G. Colangelo, The FLAG working group: Making lattice results accessible to phenomenologists, talk given at the 2009 Kaon International Conference, Tsukuba, Japan, 2009.

6. V. Lubicz, PoS LAT2009 (2009) 013.

7. C. Sachrajda, talk given at Indirect Searches for New Physics at the Time of the LHC, GGI Florence, March 2010, http://ggi-www.fi.infn.it/talks/talk1433.pdf.

8. W. J. Marciano, A. Sirlin, Phys. Rev. Lett. 71 (1993) 3629 .

9. V. Cirigliano, I. Rosell, JHEP 10 (2007) 005.

10. R. Decker, M. Finkemeier, Nucl. Phys. B438 (1995) 17.

11. M. Finkemeier, Phys. Lett. B387 (1996) 391.

12. W. J. Marciano, Phys. Rev. Lett. 93 (2004) 231803.

13. M. Knecht, H. Neufeld, H. Rupertsberger, P. Talavera, Eur. Phys. J. C12 (2000) 469.

14. A. Bazavov, et al., arXiv:0903.3598 (2009).

15. C. Allton, et al., Phys. Rev. D78 (2008) 114509.

16. JLQCD/TWQCD, PoS LAT2009 (2009) 096.

17. S. Dürr, et al., Science 322 (2008) 1224.

18. L. Del Debbio, L. Giusti, M. Luscher, R. Petronzio, N. Tantalo, JHEP 02 (2007) 082.

19. S. Aoki, et al., Phys. Rev. D79 (2009) 034503.

20. R. Baron, et al., arXiv:0911.5061 (2009).

21. K. Jansen, PoS LATTICE2008 (2008) 010.

22. S. Aoki, et al., Phys. Rev. Lett. 84 (2000) 238.

23. A. Ali Khan, et al., Phys. Rev. D65 (2002) 054505.
24. S. Aoki, et al., Phys. Rev. D68 (2003) 054502.

25. Y. Aoki, et al., Phys. Rev. D72 (2005) 114505.

26. M. Gockeler, et al., PoS LAT2006 (2006) 179.

27. B. Blossier, et al., JHEP 04 (2008) 020.

28. B. Blossier, et al., JHEP 07 (2009) 043.

29. C. Aubin, et al., Phys. Rev. D70 (2004) 114501.

30. C. Bernard, et al., PoS LAT2007 (2007) 090.

31. A. Bazavov, et al., PoS CD09 (2009) 007.

32. S. R. Beane, P. F. Bedaque, K. Orginos, M. J. Savage, Phys. Rev. D75 (2007) 094501.

33. R. Mawhinney, PoS LAT2009 (2009) 081.

34. J. Noaki, et al., PoS LAT2009 (2009) 096.

35. E. Follana, C. T. H. Davies, G. P. Lepage, J. Shigemitsu, Phys. Rev. Lett. 100 (2008) 062002.

36. C. Aubin, J. Laiho, R. S. Van de Water, PoS LATTICE2008 (2008) 105, and update from J. Laiho, talk at Chiral Dynamics 2009, Bern.

37. S. Dürr, et al., Phys. Rev. D81 (2010) 054507.

38. A. Bazavov, et al., arXiv:1004.0342 (2010).

39. M. Creutz, PoS CONFINEMENT8 (2008) 016.

40. A. S. Kronfeld, PoS LAT2007 (2007) 016.

41. M. Creutz, PoS LAT2007 (2007) 007.

42. R. Baron, et al., arXiv:1004.5284 (2010).

43. A. Sirlin, Rev. Mod. Phys. 50 (1978) 573.

44. J. Gasser, H. Leutwyler, Nucl. Phys. B250 (1985) 517.

45. H. Leutwyler, M. Roos, Z. Phys. C25 (1984) 91.

46. J. Bijnens, P. Talavera, Nucl. Phys. B669 (2003) 341.

47. M. Jamin, J. A. Oller, A. Pich, JHEP 02 (2004) 047.

48. V. Cirigliano, et al., JHEP 04 (2005) 006.

49. A. Kastner, H. Neufeld, Eur. Phys. J. C57 (2008) 541.

50. D. Becirevic, et al., Nucl. Phys. B705 (2005) 339.

51. N. Tsutsui, et al., PoS LAT2005 (2006) 357.

52. C. Dawson, T. Izubuchi, T. Kaneko, S. Sasaki, A. Soni, Phys. Rev. D74 (2006) 114502.

53. D. Brommel, et al., PoS LAT2007 (2007) 364.

54. V. Lubicz, F. Mescia, S. Simula, C. Tarantino, Phys. Rev. D80 (2009) 111502.

55. P. A. Boyle, et al., Phys. Rev. Lett. 100 (2008) 141601.

56. P. A. Boyle, et al., arXiv:1004.0886 (2010).

57. V. Bernard, E. Passemar, JHEP 04 (2010) 001.

58. V. Cirigliano, M. Giannotti, H. Neufeld, JHEP 11 (2008) 006.

59. B. Ananthanarayan, B. Moussallam, JHEP 06 (2004) 047.

60. S. Descotes-Genon, B. Moussallam, Eur. Phys. J. C42 (2005) 403.

61. V. Cirigliano, M. Knecht, H. Neufeld, H. Rupertsberger, P. Talavera, Eur. Phys. J. C23 (2002) 121.

62. V. Cirigliano, PoS KAON (2008) 007.

63. R. F. Dashen, Phys. Rev. 183 (1969) 1245.

64. J. Kambor, C. Wiesendanger, D. Wyler, Nucl. Phys. B465 (1996) 215.

65. F. Ambrosino, et al., JHEP 05 (2008) 006.

66. G. Colangelo, S. Lanz, E. Passemar, arXiv:0910.0765 (2009).

67. K. Kampf, M. Knecht, J. Novotny, M. Zdrahal, in preparation.

68. E. Abouzaid, et al., Phys. Rev. D81 (2010) 052001.

69. R. J. Hill, Phys. Rev. D74 (2006) 096006.

70. P. Franzini, PoS KAON (2008) 002.

71. V. Bernard, M. Oertel, E. Passemar, J. Stern, Phys. Lett. B638 (2006) 480.

72. C. G. Callan, S. B. Treiman, Phys. Rev. Lett. 16 (1966) 153. 


\begin{tabular}{lcccc|lcc}
\hline \hline Coeff. & $I\left(K_{e 3}^{ \pm}\right)$ & $I\left(K_{e 3}^{0}\right)$ & $I\left(K_{\mu 3}^{ \pm}\right)$ & $I\left(K_{\mu 3}^{0}\right)$ & Coeff. & $I\left(K_{\mu 3}^{ \pm}\right)$ & $I\left(K_{\mu 3}^{0}\right)$ \\
\hline$z_{0}$ & 0.14478436 & 0.14081706 & 0.09345981 & 0.09083246 & $z_{9}$ & 0.21195460 & 0.20545016 \\
$z_{1}$ & 0.50059594 & 0.48654454 & 0.30229496 & 0.29365924 & $z_{10}$ & 0.37262925 & 0.36033462 \\
$z_{2}$ & 0.69266614 & 0.67217878 & 0.48877555 & 0.47401926 & $z_{11}$ & 1.52866642 & 1.47403632 \\
$z_{3}$ & 2.39194070 & 2.31634083 & 1.80851699 & 1.75020047 & $z_{12}$ & 1.73806537 & 1.67074681 \\
$z_{4}$ & 2.35425967 & 2.27427126 & 1.84604117 & 1.78213612 & $z_{13}$ & 0.50955547 & 0.49134544 \\
$z_{5}$ & 0.79731357 & 0.77211361 & 0.60283900 & 0.58340016 & $z_{14}$ & 2.31742049 & 2.22766241 \\
$z_{6}$ & 3.13901290 & 3.03236168 & 2.46138823 & 2.37618149 & $z_{15}$ & 5.65071063 & 5.41411258 \\
$z_{7}$ & 6.74124302 & 6.49464837 & 5.40291925 & 5.20184325 & $z_{16}$ & 4.82367980 & 4.60616108 \\
$z_{8}$ & 5.13492355 & 4.93286555 & 4.17536001 & 4.00843393 & & & \\
\hline \hline
\end{tabular}

Table 15. Coefficients for the evaluation of phase space integrals from polynomial form factor parameters, for use with Eq. (65).

\begin{tabular}{lcccc|ccc}
\hline \hline Coeff. & $I\left(K_{e 3}^{ \pm}\right)$ & $I\left(K_{e 3}^{0}\right)$ & $I\left(K_{\mu 3}^{ \pm}\right)$ & $I\left(K_{\mu 3}^{0}\right)$ & Coeff. & $I\left(K_{\mu 3}^{ \pm}\right)$ & $I\left(K_{\mu 3}^{0}\right)$ \\
\hline$c_{0}$ & 0.14530227 & 0.14125098 & 0.09323787 & 0.09059706 & $c_{5}$ & 0.01797945 & 0.01724105 \\
$c_{1}$ & 0.50409859 & 0.48947133 & 0.30495081 & 0.29587728 & $c_{6}$ & 0.00545007 & 0.00515380 \\
$c_{2}$ & 1.39922105 & 1.35593685 & 0.98846227 & 0.95712982 & $c_{7}$ & 0.00128587 & 0.00119853 \\
$c_{3}$ & 3.22924652 & 3.12170948 & 2.44345185 & 2.36027254 & $c_{8}$ & 0.00025237 & 0.00023179 \\
$c_{4}$ & 6.36982500 & 6.14083977 & 6.53169253 & 4.81427789 & & & \\
\hline \hline
\end{tabular}

Table 16. Coefficients for the evaluation of phase space integrals from dispersive form factor parameters, for use with Eq. (66).

73. H. Leutwyler, private communication.

74. J. Bijnens, K. Ghorbani, arXiv:0711.0148 (2007).

75. V. Bernard, M. Oertel, E. Passemar, J. Stern, Phys. Rev. D80 (2009) 034034.

76. V. Cirigliano, J. Jenkins, M. Gonzalez-Alonso, Nucl. Phys. B830 (2010) 95.

77. R. S. Chivukula, H. Georgi, Phys. Lett. B188 (1987) 99.

78. L. J. Hall, L. Randall, Phys. Rev. Lett. 65 (1990) 2939.

79. A. J. Buras, P. Gambino, M. Gorbahn, S. Jager, L. Silvestrini, Phys. Lett. B500 (2001) 161.

80. G. D'Ambrosio, G. F. Giudice, G. Isidori, A. Strumia, Nucl. Phys. B645 (2002) 155.

81. V. Cirigliano, B. Grinstein, G. Isidori, M. B. Wise, Nucl. Phys. B728 (2005) 121.

82. Z. Han, W. Skiba, Phys. Rev. D71 (2005) 075009.

83. G. Isidori, A. Retico, JHEP 11 (2001) 001.

84. W.-S. Hou, Phys. Rev. D48 (1993) 2342.

85. A. G. Akeroyd, S. Recksiegel, J. Phys. G29 (2003) 2311.

86. V. Bernard, M. Oertel, E. Passemar, J. Stern, JHEP 01 (2008) 015.

87. Particle Data Group, C. Amsler, et al., Phys. Lett. B667 (2008) 1, and 2009 partial update for 2010 edition.

88. KLOE Collaboration, F. Ambrosino, et al., Phys. Lett. B626 (2005) 15 .

89. K. Vosburgh, et al., Phys. Rev. Lett. 26 (1971) 866.

90. KLOE Collaboration, F. Ambrosino, et al., Phys. Lett. B632 (2006) 43.

91. KTeV Collaboration, T. Alexopoulos, et al., Phys. Rev. D70 (2004) 092006.

92. NA48 Collaboration, A. Lai, et al., Phys. Lett. B645 (2007) 26.

93. KLOE Collaboration, F. Ambrosino, et al., Phys. Lett. B638 (2006) 140.

94. NA48 Collaboration, A. Lai, et al., Phys. Lett. B602 (2004) 41.

95. KLOE Collaboration, M. Adinolfi, et al., Phys. Lett. B566 (2003) 61.

96. NA48 Collaboration, A. Lai, et al., Phys. Lett. B578 (2004) 276.
97. KTeV Collaboration, A. Alavi-Harati, et al., Phys. Rev. Lett. 86 (2001) 761.

98. KTeV Collaboration, E. Abouzaid, et al., Phys. Rev. D74 (2006) 032004.

99. E. Ramberg, et al., Phys. Rev. Lett. 70 (1993) 2525.

100. Particle Data Group, S. Eidelman, et al., Phys. Lett. B592 (2004) 1, and 2005 partial update for 2006 edition.

101. KLOE Collaboration, F. Ambrosino, et al., Phys. Lett. B636 (2006) 173.

102. KLOE Collaboration, F. Ambrosino, et al., Eur. Phys. J. C48 (2006) 767.

103. NA48 Collaboration, J. R. Batley, et al., Phys. Lett. B653 (2007) 145.

104. NA48 Collaboration, A. Lai, et al., Phys. Lett. B537 (2002) 28.

105. KTeV Collaboration, A. Alavi-Harati, et al., Phys. Rev. D67 (2003) 012005.

106. V. Koptev, et al., JETP Lett. 62 (1995) 877.

107. R. Ott, T. Pritchard, Phys. Rev. D3 (1971) 52.

108. V. Fitch, C. Quarles, H. Wilkins, Phys. Rev. 140 (1965) B1088.

109. KLOE Collaboration, F. Ambrosino, et al., JHEP 01 (2008) 073.

110. KLOE Collaboration, F. Ambrosino, et al., Phys. Lett. B632 (2006) 76.

111. KLOE Collaboration, F. Ambrosino, et al., Phys. Lett. B666 (2008) 305.

112. T. Usher, et al., Phys. Rev. D45 (1992) 3961.

113. A. Vaisenberg, et al., Nucl. Phys. B115 (1976) 55.

114. L. Auerbach, et al., Phys. Rev. 155 (1967) 1505.

115. KLOE Collaboration, F. Ambrosino, et al., JHEP 02 (2008) 098.

116. A. Sher, et al., Phys. Rev. Lett. 91 (2003) 261802.

117. NA48/2 Collaboration, J. R. Batley, et al., Eur. Phys. J. C50 (2007) 329.

118. KEK-E246 Collaboration, K. Horie, et al., Phys. Lett. B513 (2001) 311.

119. KLOE Collaboration, A. Aloisio, et al., Phys. Lett. B597 (2004) 139. 
120. V. Bisi, et al., Nuovo Cim. 35 (1965) 768.

121. NA48/2 Collaboration, J. R. Batley, et al., Eur. Phys. J. C52 (2007) 1021, erratum to 117.

122. R. Wanke, communication to FlaviaNet Kaon Working Group.

123. V. Romanovsky, et al., arXiv:0704.2052 (2007).

124. Particle Data Group, W.-M. Yao, et al., J. Phys. G33 (2006) 1, and 2007 partial update for 2008 edition.

125. F. Lobkowicz, et al., Phys. Rev. 185 (1969) 1676.

126. I.-H. Chiang, et al., Phys. Rev. D6 (1972) 1254.

127. O. Yushchenko, communication to FlaviaNet Kaon Working Group.

128. KLOE Collaboration, F. Ambrosino, et al., Phys. Lett. B636 (2006) 166.

129. KTeV Collaboration, T. Alexopoulos, et al., Phys. Rev. D70 (2004) 092007.

130. NA48 Collaboration, A. Lai, et al., Phys. Lett. B604 (2004) 1.

131. O. Yushchenko, et al., Phys. Lett. B589 (2004) 111.

132. KLOE Collaboration, F. Ambrosino, et al., JHEP 12 (2007) 105.

133. NA48 Collaboration, A. Lai, et al., Phys. Lett. B647 (2007) 341.

134. O. Yushchenko, et al., Phys. Lett. B581 (2004) 31.

135. M. Antonelli, et al., arXiv:0907.5386 (2009).

136. M. Testa (for the KLOE Collaboration), arXiv:0805.1969 (2008).

137. S. Fubini, G. Furlan, Lett. Nuovo Cim. 3S1 (1970) 168.

138. M. J. Ramsey-Musolf, S. Su, S. Tulin, Phys. Rev. D76 (2007) 095017.

139. M. Davier, A. Hocker, Z. Zhang, Rev. Mod. Phys. 78 (2006) 1043.

140. J. C. Hardy, I. S. Towner, Phys. Rev. C79 (2009) 055502.

141. W. J. Marciano, A. Sirlin, Phys. Rev. Lett. 96 (2006) 032002.

142. I. Towner, J. Hardy, Phys. Rev. C77 (2008) 025501.

143. M. Bona, et al., arXiv:0908.3470 (2009). 